\title{
p63 uses a switch-like mechanism to set the threshold for induction of apoptosis
}

\author{
Jakob Gebel ${ }^{1,8}$, Marcel Tuppi ${ }^{1,7,8} \bowtie$, Apirat Chaikuad ${ }^{2,8}$, Katharina Hötte ${ }^{3}$, Martin Schröder ${ }^{2}$, \\ Laura Schulz ${ }^{4}$, Frank Löhr', Niklas Gutfreund', Franziska Finke', Erik Henrich', Julija Mezhyrova', \\ Ralf Lehnert ${ }^{5}$, Francesco Pampaloni ${ }^{3}$, Gerhard Hummer ${ }^{4,6}$, Ernst H. K. Stelzer ${ }^{3}$, Stefan Knapp $\mathbb{1}^{2}$ and \\ Volker Dötsch $\mathbb{1}^{1 凶}$
}

The p53 homolog TAp63 $\alpha$ is the transcriptional key regulator of genome integrity in oocytes. After DNA damage, TAp63 $\alpha$ is activated by multistep phosphorylation involving multiple phosphorylation events by the kinase CK1, which triggers the transition from a dimeric and inactive conformation to an open and active tetramer that initiates apoptosis. By measuring activation kinetics in ovaries and single-site phosphorylation kinetics in vitro with peptides and full-length protein, we show that TAp63 $\alpha$ phosphorylation follows a biphasic behavior. Although the first two CK1 phosphorylation events are fast, the third one, which constitutes the decisive step to form the active conformation, is slow. Structure determination of CK1 in complex with differently phosphorylated peptides reveals the structural mechanism for the difference in the kinetic behavior based on an unusual CK1/TAp63 $\alpha$ substrate interaction in which the product of one phosphorylation step acts as an inhibitor for the following one.

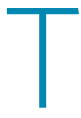
he reproductive lifespan of women is determined by the primordial follicle (PF) reserve. PFs consist of primary oocytes surrounded by a single layer of granulosa cells. The depletion of the PF reserve has been identified as the major cause of premature ovarian insufficiency (POI). In female patients suffering from cancer, sickle cell anemia or certain autoimmune diseases, treatment with chemotherapeutic drugs and/or irradiation can deplete the ovarian reserve, resulting in $\mathrm{POI}^{1,2}$. Fewer than 10 DNA double-strand breaks (DSBs) per oocyte are sufficient to eliminate the entire PF reserve in mice ${ }^{3}$. In humans, the $\mathrm{LD}_{50}$ (lethal dose to $50 \%$ of individuals tested) total body irradiation dosage for loss of the PF reserve was extrapolated to be less than $2 \mathrm{~Gy}$, while the typical total body irradiation dose for acute leukemia patients is $12 \mathrm{~Gy}$ (refs. ${ }^{4,5}$ ). The loss of the PF reserve causes infertility and breakdown of the endocrine function of the ovary, resulting in health impairments such as osteoporosis ${ }^{6}$. In contrast to the PFs, growing follicles are unaffected by a low dosage of irradiation. The decisive difference between these oocyte types is the expression of TAp63 $\alpha$, a p53 ortholog, in $\mathrm{PFs}^{3,7}$. The high expression level of pro-apoptotic TAp63 $\alpha$ requires very tight regulation of its activity to avoid cell death of uncompromised oocytes. In contrast to all other members of the p53 family, which form tetramers, TAp63 $\alpha$ adopts a closed, inactive and only dimeric conformation ${ }^{8}$. DNA damage leads to phosphorylation of TAp63 $\alpha$, which disrupts the autoinhibitory complex and triggers the formation of an active and tetrameric state $^{9}$. Recently, we and others have identified the kinases involved in this process, mapped the phosphorylation sites and described the mechanism of the activation process. The first step is the activation of checkpoint kinase 2 (CHK2) or checkpoint kinase 1(CHK1) by ataxia telangiectasia mutated kinase $(\mathrm{ATM})^{10}$. Activated $\mathrm{CHK} 2$ phosphorylates TAp63 $\alpha$ on $5582^{11}$, which renders TAp63 $\alpha$ a substrate for kinase $\mathrm{CK} 1^{12}$. CK1 usually requires pre-phosphorylated substrates with the sequence $\mathrm{pS} / \mathrm{T}-\mathrm{x}-\mathrm{x}-\mathrm{S} / \mathrm{T}$, where $\mathrm{pS} / \mathrm{T}$ is a phosphorylated serine or threonine $\mathrm{e}^{13-15}$. In TAp63 $\alpha$, CK1 adds four phosphate groups in a sequential manner, with each newly added phosphate acting as the priming site for the following phosphorylation event. The accumulation of negative charge leads to disruption of the autoinhibitory complex through electrostatic repulsion ${ }^{12}$. We have shown previously that the dimeric state of TAp63 $\alpha$ constitutes a kinetically trapped high-energy state ${ }^{16}$. Consequently, activation follows a spring-loaded mechanism that explains the high sensitivity of oocytes to DNA damage. This activation mechanism has to be adjusted to a certain level of damage that on the one hand must be sufficiently low to protect the integrity of the genetic pool but on the other hand tolerant enough not to endanger the reproductive capacity. A tight dose-response curve has indeed been observed in oocytes of $\mathrm{P} 4$ mice. Although most oocytes survived irradiation with $0.1 \mathrm{~Gy}$ (approximately three DSBs per cell), virtually all primary oocytes were eliminated by $0.45 \mathrm{~Gy}(\sim 10 \mathrm{DSB} \text { per cell })^{3}$. Such switch-like processes are often based on the integration of two different and independent signals. Accidental activation is thus suppressed, as the likelihood of activation is the product of two small probabilities $^{17,18}$. The activation of TAp63 $\alpha$ is based on phosphorylation by two kinases, which, in principle, could provide such a sigmoidal activation. However, both act in a sequentially dependent manner and CK1 kinases are thought to be constitutively active ${ }^{15}$. These properties would prevent switch-like activation and make the initiation of apoptosis dependent only on CHK2, with potentially detrimental consequences for the safety of oocytes. Here, we investigate how TAp63 $\alpha$ converts a graded stress response into a

IInstitute of Biophysical Chemistry and Center for Biomolecular Magnetic Resonance and Cluster of Excellence Macromolecular Complexes (CEF), Goethe University, Frankfurt am Main, Germany. ${ }^{2}$ Institute of Pharmaceutical Chemistry, Goethe University, Frankfurt am Main, Germany. ${ }^{3}$ Physikalische Biologie, Buchmann Institute for Molecular Life Sciences (BMLS), Goethe University, Frankfurt am Main, Germany. ${ }^{4}$ Department of Theoretical Biophysics, Max Planck Institute of Biophysics, Frankfurt am Main, Germany. ${ }^{5}$ Mathezentrum, Goethe University, Frankfurt am Main, Germany. ${ }^{6}$ Institute of Biophysics, Goethe University, Frankfurt am Main, Germany. ${ }^{7}$ Present address: The Francis Crick Institute, London, UK. ${ }^{8}$ These authors contributed equally: Jakob Gebel, Marcel Tuppi, Apirat Chaikuad.凶e-mail: marcel.tuppi@crick.ac.uk; vdoetsch@em.uni-frankfurt.de 
a

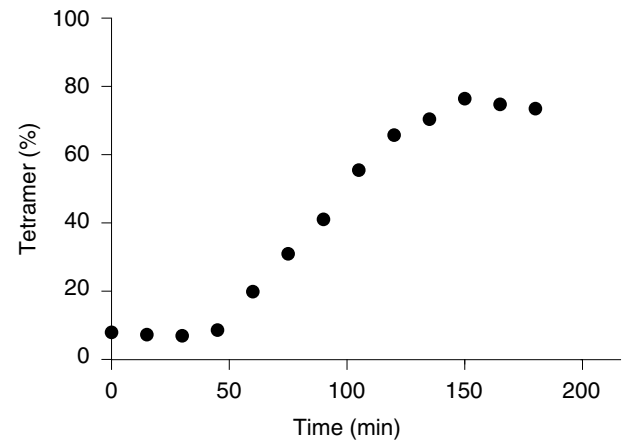

c
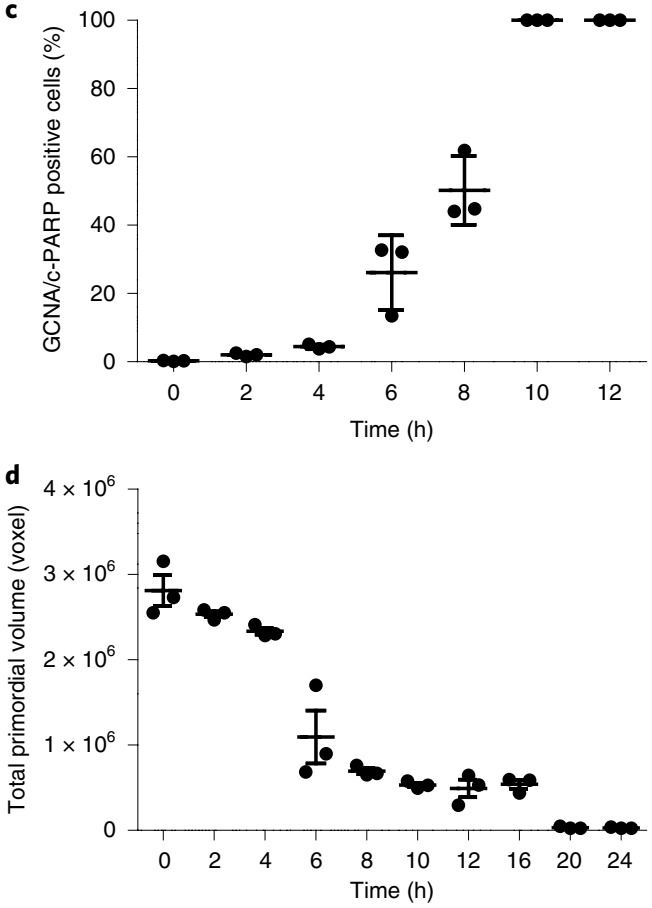
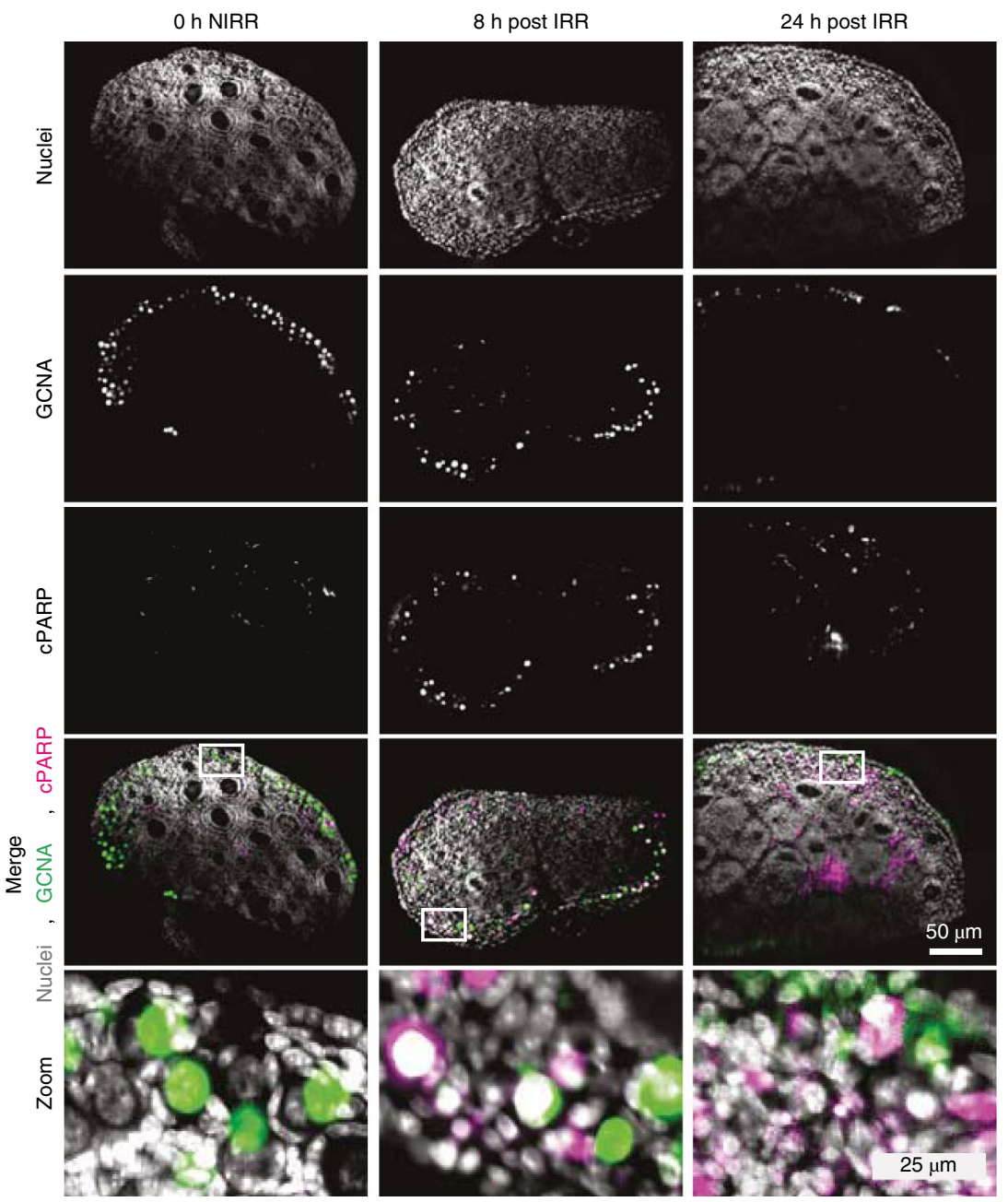

Fig. 1 | TAp63 $\alpha$-mediated PF death follows an overall sigmoidal kinetics. a, Whole mouse ovaries were ex vivo gamma-irradiated with $0.5 \mathrm{~Gy}$ or left untreated. The oligomeric state was analyzed as a function of time by blue native polyacrylamide gel electrophoresis (BN-PAGE) and the dimeric and tetrameric fractions were analyzed densitometrically. $\mathbf{b}$, Representative single-plane images of whole-mount 3D ovary stainings using 4',6-diamidino2-phenylindole (DAPI) (nuclei), GCNA and cleaved PARP (c-PARP) at the indicated time points after the 0.5-Gy gamma irradiation (IRR) and of untreated ovaries. Three individual ovaries from three different animals were imaged. Scale bar, $50 \mu \mathrm{m}$. For the three enlarged sections the white box in the lower right panel corresponds to $25 \mu \mathrm{m}$ in length. c, Ratio of c-PARP-positive GCNA-positive cells of whole ovaries after 0.5-Gy gamma irradiation using whole-mount 3D ovary staining. d Time-dependent GCNA-positive cells, quantified in voxels, after 0.5 Gy gamma irradiation using whole-mount 3D ovary staining. Data in a were repeated once with a different time frame and are shown in Supplementary Fig. $1 \mathrm{~b}$. In $\mathbf{c}$ and $\mathbf{d}$, data are presented as mean \pm s.d. $(n=3$ individual ovaries from different animals). See Supplementary Fig. 1.

switch-like activation mechanism. We show that the CK1 phosphorylation events follow a biphasic kinetic, with the third one, which is essential for formation of the open and active conformation, being the slowest. Crystal structure determination revealed an unusual kinase-product state explaining the observed kinetics. We speculate that this difference in the kinetics sets the threshold of DNA damage required for the elimination of damaged oocytes.

\section{Results}

Apoptosis follows a sigmoidal time response. We set out to measure the time-dependent TAp63 $\alpha$ activation in whole mouse ovaries following DNA damage as a response to gamma irradiation. The tetramerization kinetics of TAp63 $\alpha$ upon gamma irradiation followed a sigmoidal time response, with virtually all TAp63 $\alpha$ converted into the active tetrameric state within $2 \mathrm{~h}$ (Fig. 1a and Supplementary Figs. 1a,b and 2). Activation can be suppressed using selective inhibitors of ATM, CHK2 and CK1, as shown previously for cisplatin or doxorubicin (Supplementary Fig. 1b) ) $^{12,16,19}$. To investigate the time-dependent induction of apoptosis, we measured the ratio of germ cell nuclear acidic peptidase (GCNA) ${ }^{20}$ positive and cleaved poly(ADP-ribose)-polymerase 1 (PARP1) double-positive cells in whole mouse ovaries using our three-dimensional (3D) staining and light sheet-based fluorescence microscopy combined with semi-automated segmentation method (Fig. 1b and Supplementary Fig. 1c) ${ }^{12,21-23}$. Cleaved PARP1 was detected starting from $4 \mathrm{~h}$ after irradiation and reached $100 \%$ in the remaining PFs by $10 \mathrm{~h}$, showing an overall sigmoidal transition (Fig. 1c). A sigmoidal transition was also evident when monitoring the decline of the GCNA signal representing the total number of remaining primary oocytes (Fig. 1d).

Phosphorylation of the third CK1 site is the slowest. The correlation between the sigmoidal time-response patterns for TAp63 $\alpha$ 
a

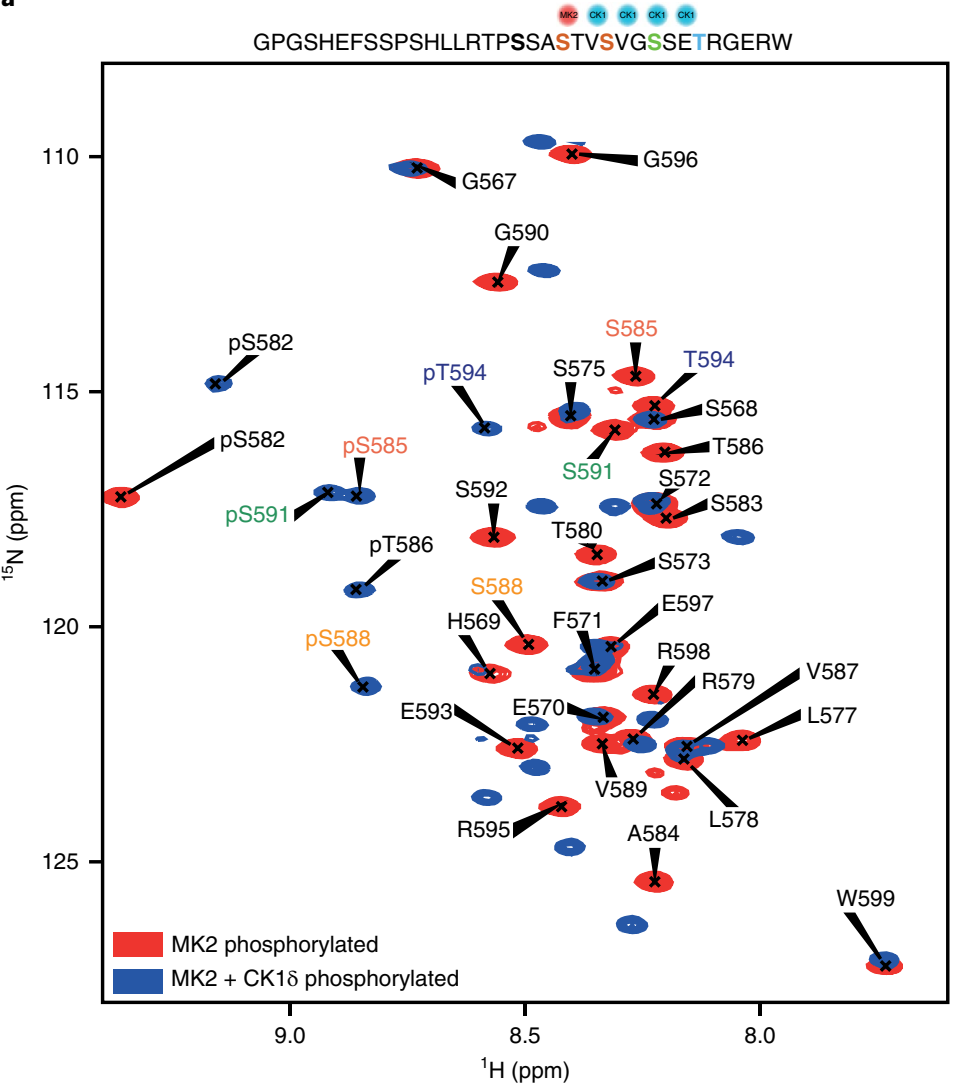

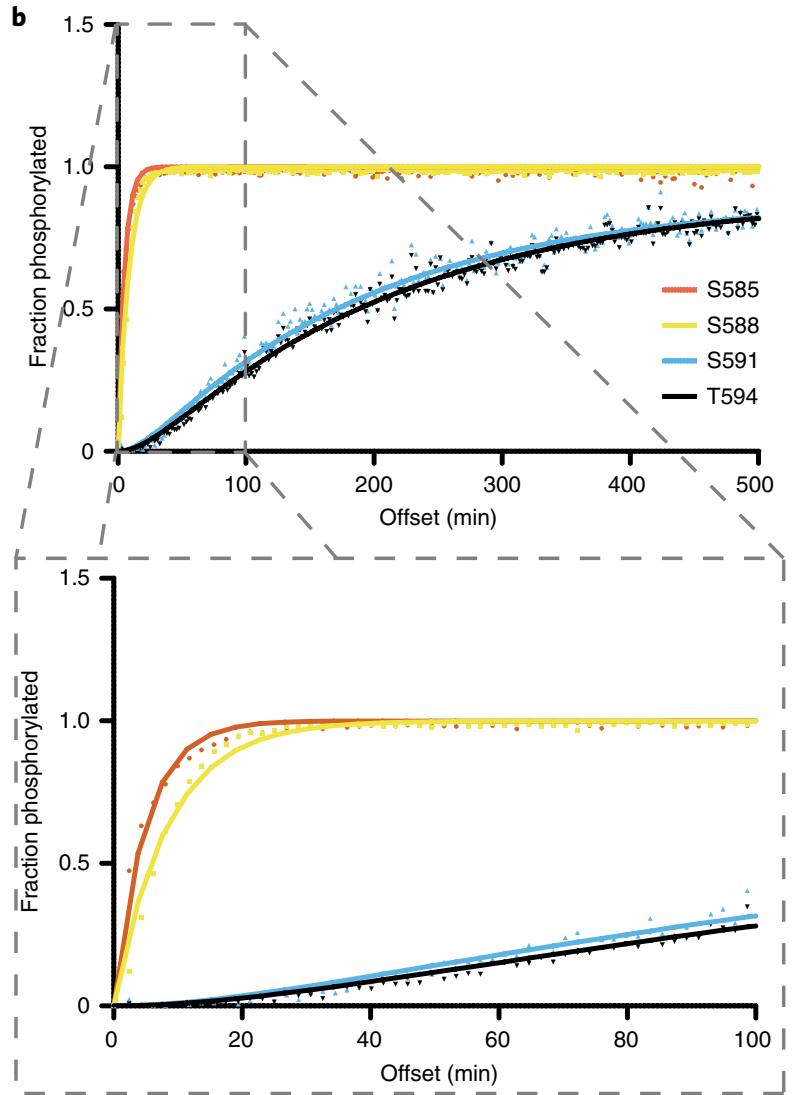

Fig. 2 | The third CK1 phosphorylation is the slowest step in the phosphorylation of a p63-derived peptide (PAD). a, Single-site phosphorylation kinetics, measured by NMR spectroscopy. An overlay is shown of MK2 pre-phosphorylated (red) and CK1 phosphorylated (blue) [ $\left.{ }^{15} \mathrm{~N},{ }^{1} \mathrm{H}\right]-\mathrm{HSQC}$ spectra of the PAD peptide, representing the starting and end points. The analyzed peptide sequence is shown above the spectra. $\mathbf{b}$, Quantitative evaluation of the phosphorylation kinetics of the four CK1 sites. Bottom: zoomed-in graph focusing on the initial reaction, showing that the third and fourth sites are modified after the first two sites are almost 100\% phosphorylated. The peptide kinetics were measured twice under identical conditions. See also Extended Data Fig. 1 and Supplementary Table 1.

tetramerization and induction of apoptosis suggests that the activation mechanism of TAp63 $\alpha$ is the decisive process. Previously, we have shown that the initial phosphorylation of 5582 by CHK2 is necessary but does not result in active TAp63 $\alpha$. This phosphorylation serves as the priming event for recruiting CK1, which sequentially adds phosphate groups at four consecutive sites: S585, S588, S591 and T594 $4^{12}$. We characterized each phosphate's importance for the activation mechanism by mutating individual residues to alanine and measuring the tetramerization kinetics, demonstrating that the last phosphorylation event, T594, is dispensable for activation. However, mutating S591 abrogates the conversion of TAp63 $\alpha$ to a tetramer, showing that phosphorylation of S591 is the "point of no return" ${ }^{12}$. This observation raised the question of how phosphorylation of the three CK1 sites, S585, S588 and S591, can provide a switch-like activation mechanism. Detailed theoretical and experimental studies have shown that multisite phosphorylation can lead to ultrasensitivity ${ }^{24-26}$. A switch-like activation can be achieved if multisite phosphorylation occurs in a distributive mode ${ }^{27}$. Besides, a distributive process would allow the interference of a phosphatase, while a processive mode would result in continuous phosphorylation of all sites. To investigate the mode of action, we analyzed the phosphorylation kinetics of individual sites within a peptide that included all phosphorylation sites for CHK2 and CK1 (phosphorylation activation domain, PAD) located N-terminal to the transactivation inhibitory domain (TID) $)^{28,29}$. For these investigations, we used NMR spectroscopy ${ }^{30-36}$ based on a gradient-selected Band-selective excitation short-transient transverse relaxation-optimized spectroscopy
(BEST-TROSY) pulse sequence ${ }^{37}$, allowing us to restrict the measurement time of each 2D spectrum to $120 \mathrm{~s}$. Phosphorylation of S582 by MapKap kinase 2 (MK2), which recognizes the same phosphorylation sequence as $\mathrm{CHK} 2$, resulted in new resonance in the TROSY spectrum and loss of the original S582 signal (Extended Data Fig. 1a). MK2 can be produced easily in bacteria and is therefore a convenient surrogate for CHK2. The phosphorylation kinetics of this pre-phosphorylated peptide by CK1 $\delta$ (substrate:kinase ratio 2,000:1) showed a biphasic behavior. Phosphorylation of S585 and S588 occurred very quickly with almost indistinguishable kinetics, corresponding to either a processive mode or to a distributive mode with very fast product release and substrate rebinding. We observed a stark contrast in the subsequent phosphorylation of S591, with an $\sim 23$-fold slower rate (Fig. 2a,b and Supplementary Table 1). The increase in the concentration of the triple phosphorylated peptide (pS582, pS585, pS588; PAD-3P) beyond the kinase concentration suggests that CK1 must have dissociated from the PAD-3P product peptide to phosphorylate S585 and S588 in free PAD-1P and PAD-2P peptides. Only when the supply of pS582 mono-phosphorylated peptide is exhausted did S591 and T594 become substrates for CK1. To distinguish between a distributive and a processive mechanism for S585 and S588 as well, we repeated the experiments at a lower kinase concentration (substrate-to-kinase ratio of 20,000:1). Under these conditions, phosphorylation of S588 is slower than phosphorylation of S585 with the concentration of the dual phosphorylated peptide (pS582, pS585; PAD-2P) exceeding the kinase concentration (Extended Data Fig. 1b). We also measured the phosphorylation 
a
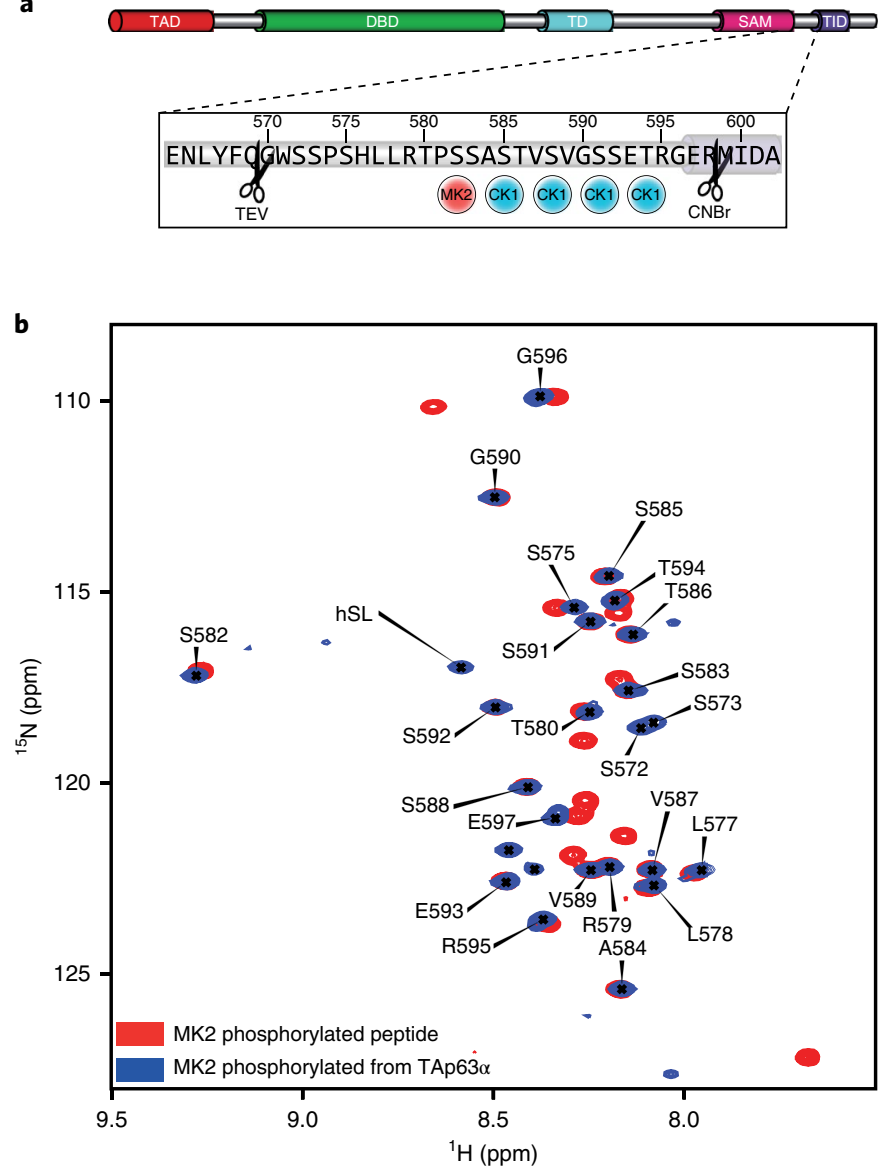

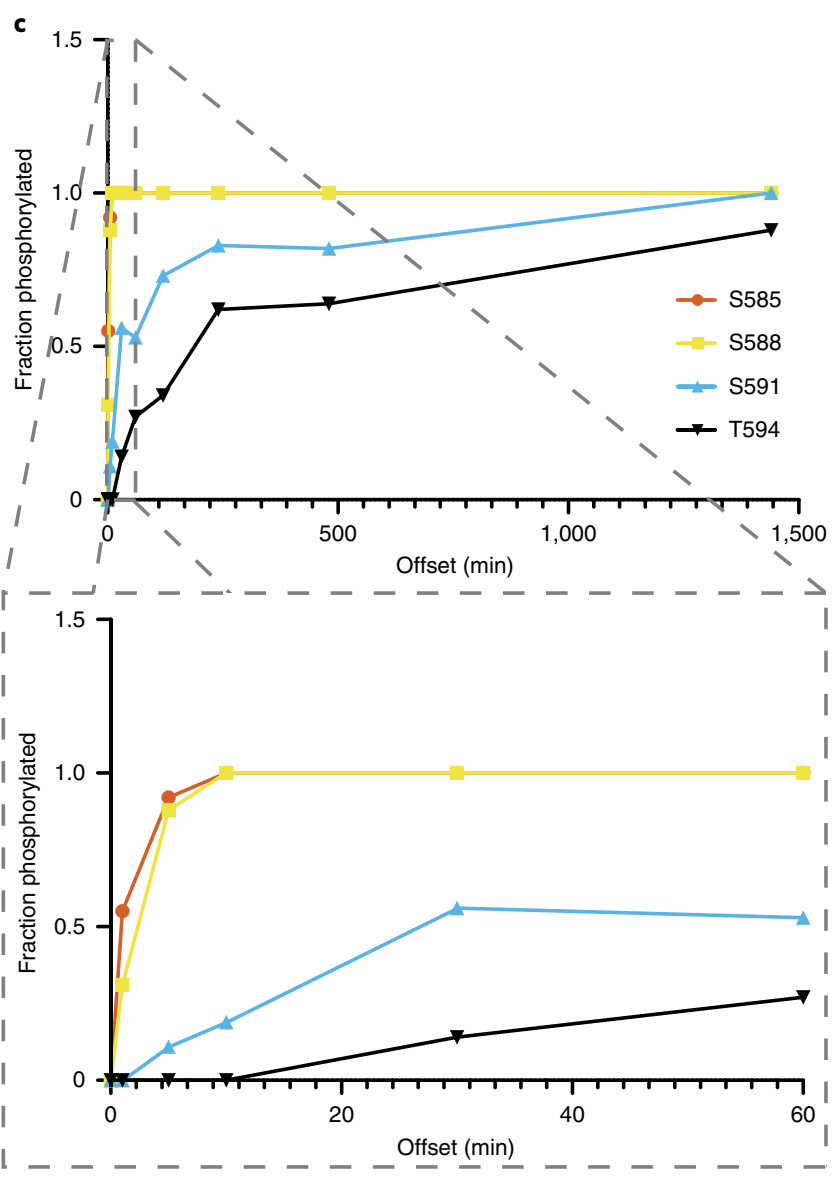

d

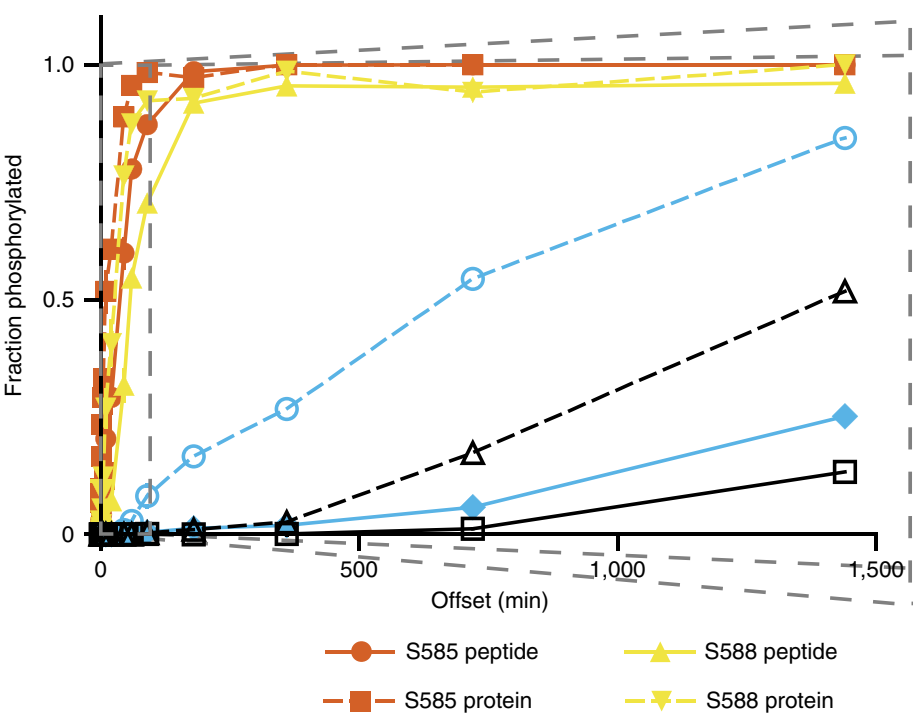

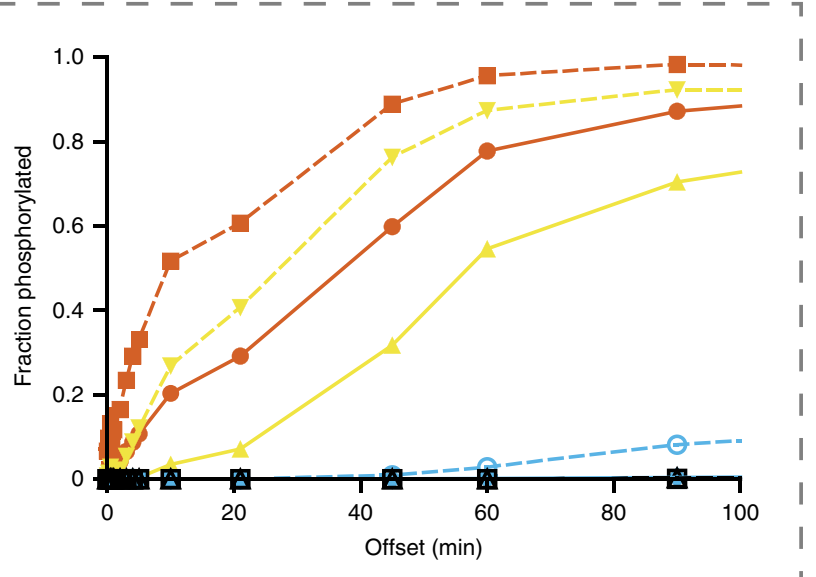

$$
\begin{array}{ll}
- \text { S591 peptide } & \square-\text { T594 peptide } \\
-\ominus-\text { S591 protein } & -\mathbf{A}-\text { T594 protein }
\end{array}
$$

Fig. 3 | The third CK1 phosphorylation is the slowest step in the TAp63 $\alpha$ phosphorylation and constitutes the 'point of no return'. a, Schematic representation of the domain structure of TAp63 $\alpha$ showing the location of the PAD peptide and the sites used for cleavage of the peptide by TEV protease and $\mathrm{CNBr}$. b, Overlay of $\left[{ }^{15} \mathrm{~N},{ }_{1} \mathrm{H}\right]-\mathrm{HSQC}$ spectra of MK2 pre-phosphorylated PAD peptide (red) and MK2 pre-phosphorylated PAD peptide cleaved from full-length TAp63 $\alpha$ (blue). hSL indicates the position of the homo-serine-lactone resonance position, created during the CNBr cleavage. $\mathbf{c}$, Evaluation of the phosphorylation kinetics of the four CK1 sites in full-length TAp63 $\alpha$. Bottom: zoomed-in graph (dashed lines) focusing on the initial 60 min. $\mathbf{d}, \mathrm{MS}$-based kinetics with a sample containing both peptide and TAp63 $\alpha$ polypeptide chains in a 2:1 ratio to account for the dimeric state of TAp63 $\alpha$. NMR kinetics were measured twice with different kinase:TAp63 $\alpha$ ratios (Supplementary Fig. 4g). Right: zoomed-in graph (dashed lines) focusing on the initial 100 min. The MS-based kinetics were measured once.

kinetics of S591 and T594, starting with the PAD-3P peptide, demonstrating that modification of T594 similarly follows a distributive mechanism (Extended Data Fig. 1c). We further confirmed by mutational analysis that CK1 phosphorylates the peptide in a sequential manner, with each new phosphate acting as the priming site for the following phosphorylation step (Extended Data 

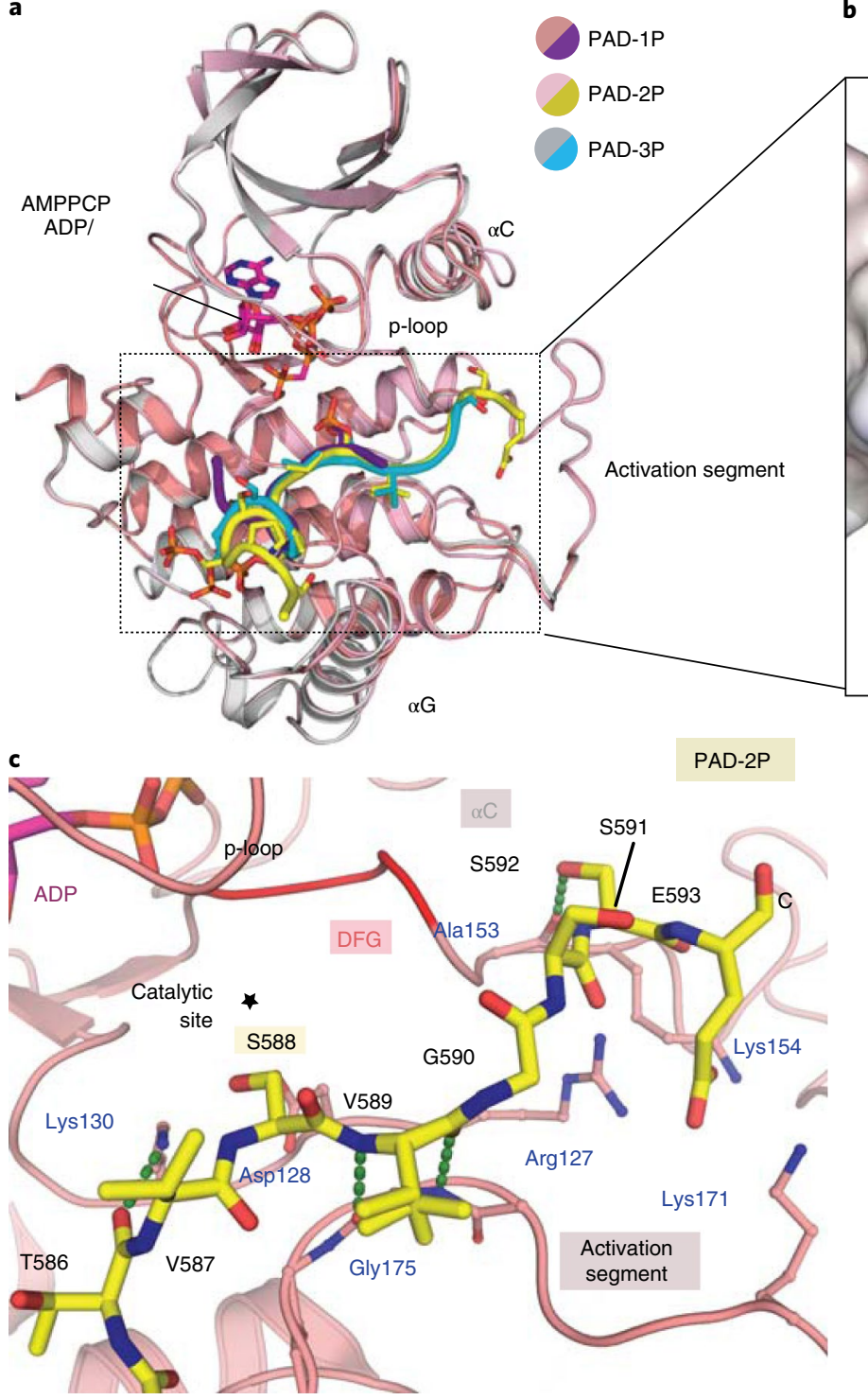

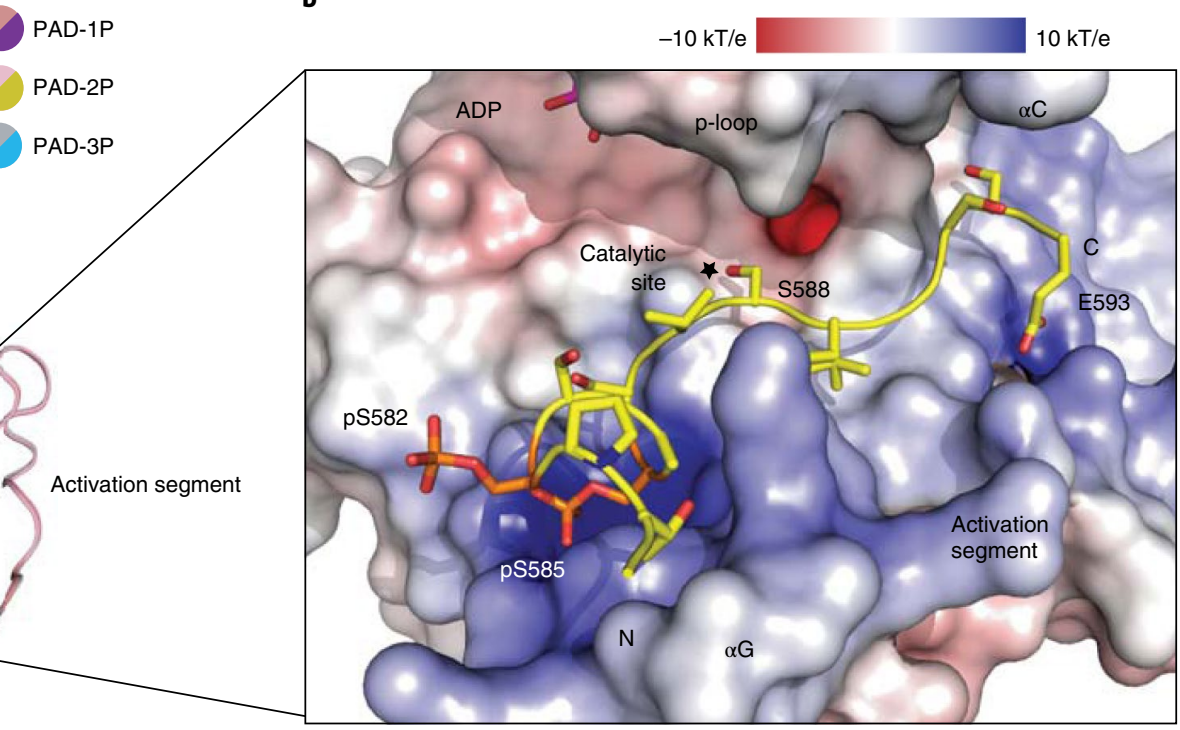

d

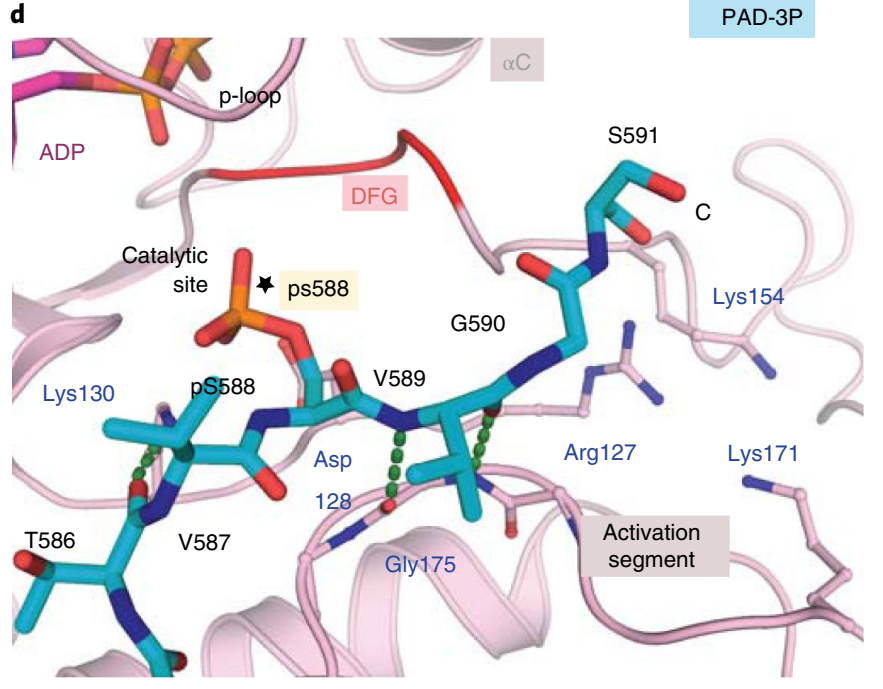

Fig. 4 | Crystal structures of CK1 in complexes with different PAD peptides. a, Superimposition of all complexed structures demonstrates a similar binding conformation of the PAD peptides within the kinase. Three PAD peptides, colored differently as indicated, harbor different phosphorylation states, including PAD-1P containing single phosphorylated S582, PAD-2P with double phosphorylations on S582 and S585, and PAD-3P with triple phosphorylations on S582, S585 and S588. b. Electrostatic potential on the kinase surface at the substrate peptide binding sites reveals both charge and shape complementarity for the PAD-2P peptide accommodation. $\mathbf{c}, \mathbf{d}$, Detailed interactions between the PAD-2P or PAD-3P and the kinase are highly similar, despite representing different states of substrate- and product-bound complexes, respectively. DFG, Asp-Phe-Gly triad; HRD, His-Arg-Asp triad; p-loop, phosphate-binding loop, also known as the glycine-rich loop. CK1 amino acids are labeled in three-letter code (blue) and PAD residues are labeled in one-letter code (black). See Extended Data Fig. 3 and Supplementary Table 2.

Fig. 1d-f). Based on these observations, we conclude that (1) the mode of phosphorylation of the entire segment is sequential and distributive and (2) S585 and S588 act as a buffer that delays phosphorylation of S591 and T594. Interestingly, phosphorylation of S591 is the point of no return for the activation, and these kinetic experiments demonstrate that this critical phosphorylation is the slow step occurring in a distributive mode. In the same time frame as the phosphorylation of S591 and T594, the non-consensus sequence phosphorylation of T586 appeared. The T586A mutant, however, showed no difference in the phosphorylation kinetics (Extended Data Fig. 1g).

The observed biphasic behavior could be a property of the p63 sequence, of CK1 substrate recognition or a mixture of both. The CK1 family recognizes several hundred confirmed or suggested substrates ${ }^{15}$, including some containing several phosphorylatable residues (Supplementary Fig. 3a). To investigate if CK1 $1 \delta$ can add more than two phosphate groups without a substantial delay, we chose yes-associated protein 1 (YAP1), which contains a phosphodegron sequence ${ }^{38}$. Measuring the kinetics of the three CK1 phosphorylation events demonstrated similar kinetics for all three sites, suggesting that CK1 phosphorylates the sequence in YAP1, and possibly other proteins, without a distinct biphasic mechanism (Supplementary Fig. 3b-e).

The kinetics of TAp63 $\alpha$ mirrors that of peptides. The phosphorylation kinetics of the isolated PAD peptide might, however, be different from the kinetics of the peptide within the closed and dimeric full-length TAp63 $\alpha$, because this sequence is surrounded by secondary structure elements and folded domains (Fig. 3a). We measured the phosphorylation kinetics with ${ }^{15} \mathrm{~N}$-labeled full-length dimeric TAp63 $\alpha$. To analyze the phosphorylation kinetics by NMR, 

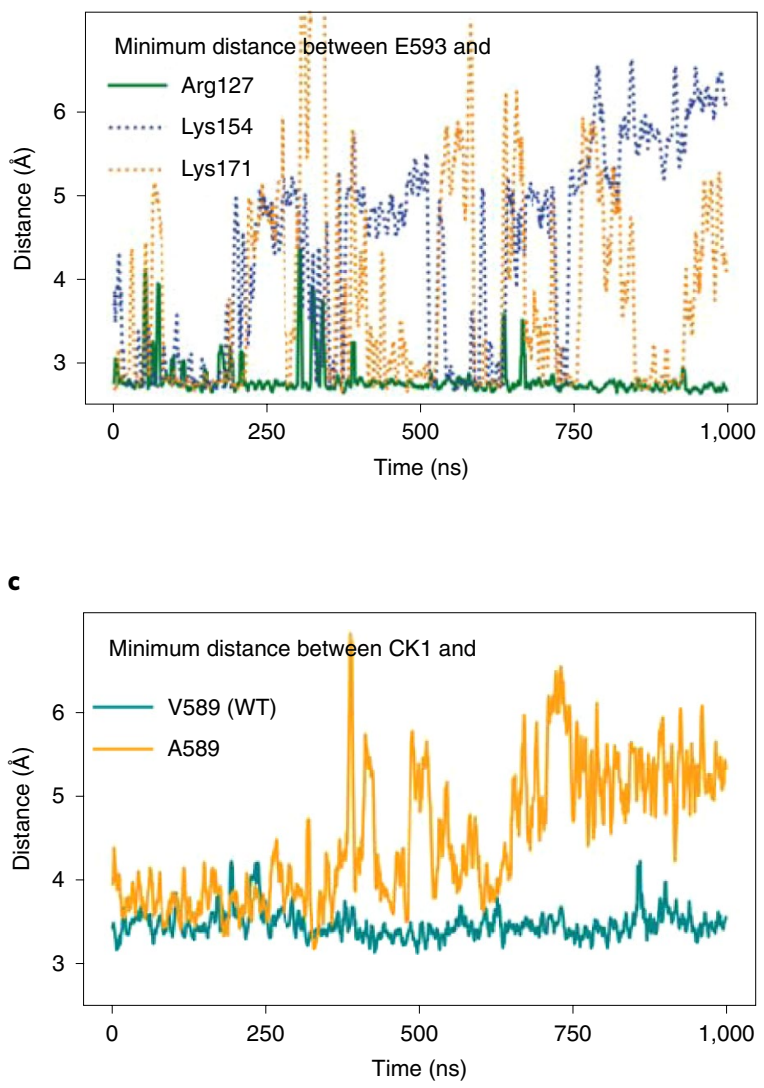

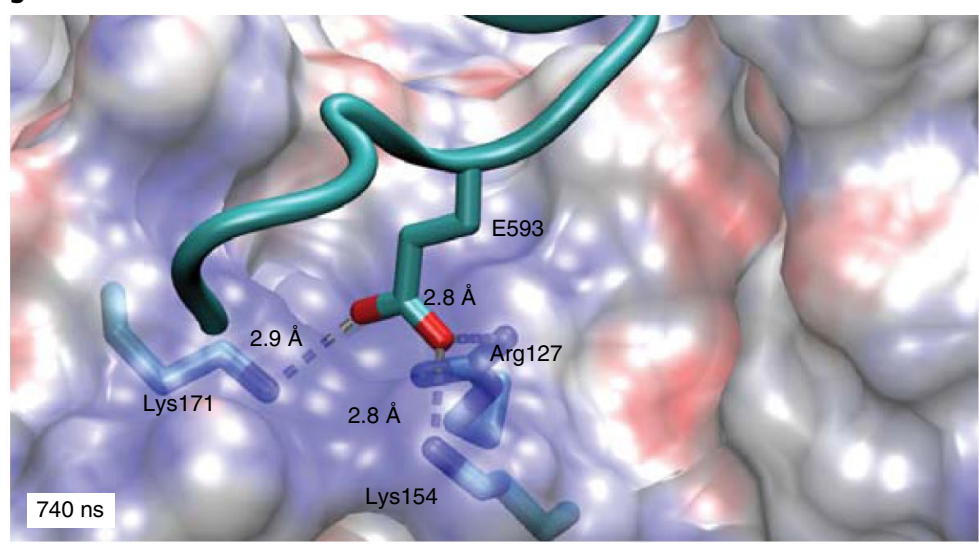

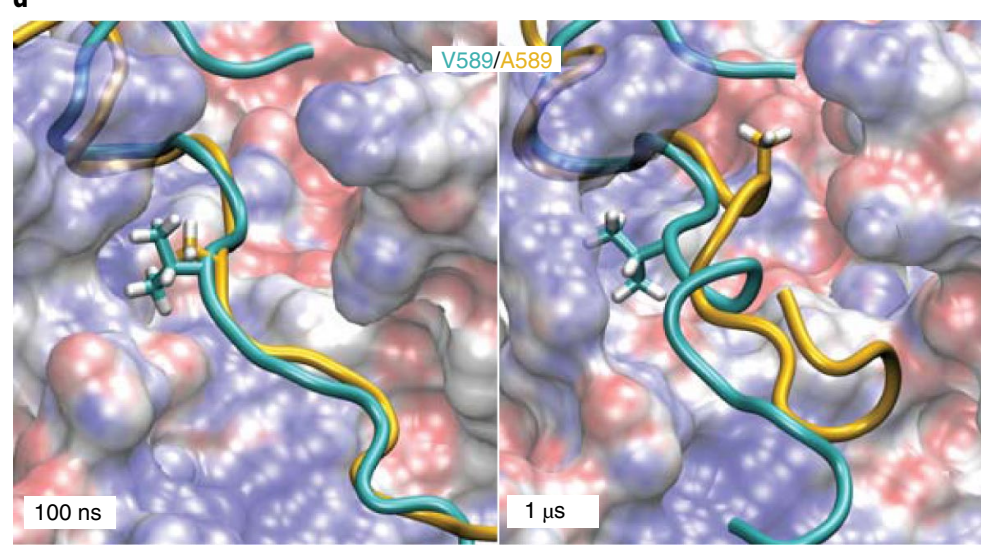

Fig. 5 | MD simulations indicate that E593 and V589 of the p63 peptide are important for interaction with CK1. a, E593 is pinned down by salt bridges with CK1. Minimum heavy-atom distances of E593 to Arg127 and Lys154 are shown as a function of time. E593 forms a salt bridge with Lys154 (distance $<3 \AA$ ), and binds transiently to Lys154. b, Representative snapshot at 740 ns, zooming in on the C-terminal region of the p63 peptide. CK1 is shown as a transparent electrostatic surface (blue/red for positive/negative charge), and the p63 peptide is represented as a cyan cartoon. Residues E593, Arg127 and Lys154 are highlighted. The minimum distances between E593 and the basic residues are indicated. c, Mutation of V589 to A589 enhances the flexibility of the p63 peptide. Minimum heavy-atom distances of CK1 to V589 and A589 in wild type and mutant p63 peptides are shown as a function of time. V589 remains adhered to the CK1 protein surface, whereas A589 becomes solvated and moves away from the CK1 protein surface. $\mathbf{d}$, Snapshots at $100 \mathrm{~ns}$ and $1 \mu \mathrm{s}$, zooming in on the $\mathrm{C}$-terminal region of the $\mathrm{p} 63$ peptide. CK1 structures are superimposed. CK1 from the wild-type simulation is represented as in $\mathbf{b}$, and the p63 peptide is shown in cartoon representation (cyan, wild type; yellow, V589A) with V589 and A589 highlighted. CK1 amino acids are labeled in three-letter code and PAD residues in one-letter code. Results are shown for the longer p63 construct. See Extended Data Fig. 4 for the capped and shortened $\mathrm{p} 63$ construct.

we stopped the reaction by adding EDTA and CK1 inhibitor after specific time points. Subsequently, we isolated the PAD peptide first by cleaving with tobacco etch virus (TEV) protease. After isolating the resulting $\mathrm{C}$-terminal fragment, the PAD was cleaved from the TID using CNBr (Supplementary Fig. 4a-f).

The phosphorylation kinetics of the PAD peptide and the PAD derived from the dimeric TAp63 $\alpha$ showed a similar pattern. S585 and S588 were phosphorylated quickly, while phosphorylation of S591 and T594 was significantly slower (Fig. 3b,c). To investigate if a further increase in the kinase concentration eliminates the biphasic behavior, we measured the phosphorylation kinetics in full-length TAp63 $\alpha$ at a concentration ratio of 1:100. In these conditions, phosphorylation of S585 and S588 was virtually complete before measurement of the first 2D NMR spectrum ( $2.5 \mathrm{~min})$. In contrast, the kinetics of S591 and T594 phosphorylation proceeded still slower. These experiments also confirmed that phosphorylation of T594 is slower than phosphorylation of S591, supporting a sequential distributive mechanism for the entire sequence (Supplementary Fig. 4g).
Initially, we conducted these experiments with the full-length protein because we wanted to investigate potential steric hindrance effects. Surprisingly, phosphorylation of the third CK1 site is not slower, but is faster within the full-length protein (Figs. $2 \mathrm{~b}$ and $3 \mathrm{c}$ ). This might be a consequence of the dimeric state of TAp63 $\alpha$ presenting two binding sites for CK1 per molecule, which might increase the local concentration of $\mathrm{CK} 1$ and thus accelerate phosphorylation. To investigate this effect, we measured the phosphorylation kinetics of the PAD sequence in the peptide versus full-length TAp63 $\alpha$ (T586A) using a MS approach. We mixed TAp63 $\alpha$ and a twofold higher concentration of the ${ }^{15} \mathrm{~N}$-labeled peptide and added CK1. After the indicated time points, the reaction was stopped and both the TAp63 $\alpha$ derived and the ${ }^{15} \mathrm{~N}$-labeled PAD peptides were purified as described above and analyzed using a MALDI-LTQ (matrix-assisted laser desorption/ionization-linear ion trap) Orbitrap XL mass spectrometer. The results of this 'competition experiment' confirmed that all phosphorylation events in the protein are faster than in the peptide (Fig. 3d). However, rate differences between S585/S588 and S591 phosphorylation persist, the latter being slower in both cases. 

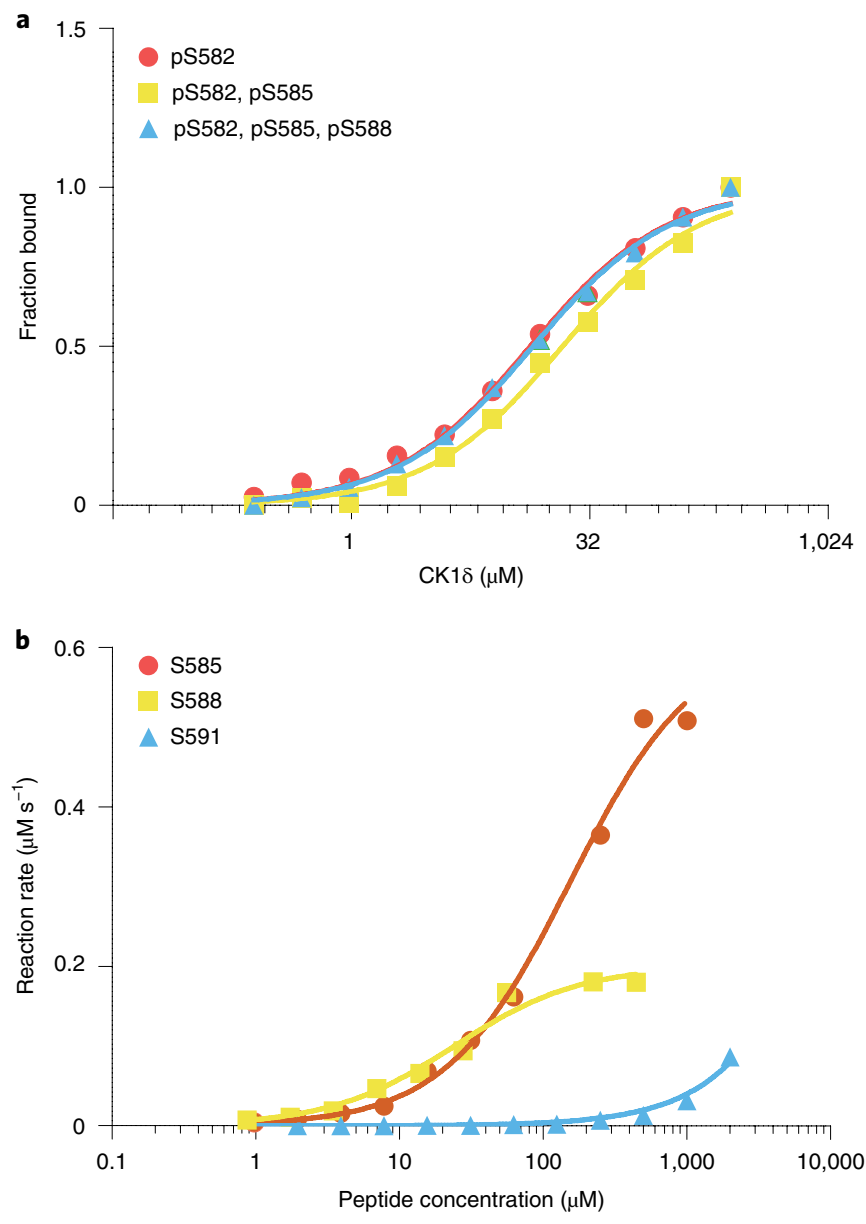

Fig. 6 | The interaction affinity between CK1 and PAD peptides does not depend on the phospho-state, but $K_{\mathrm{M}}$ values differ by more than an order of magnitude. $\mathbf{a}$, The binding affinity of differently phosphorylated peptides varies by a factor of only $~ 1.3$, with the triple phosphorylated peptide having the highest affinity. The curves show one experiment out of a series of three replicates per peptide. See Extended Data Fig. 5a and Supplementary Table 3. Values in Supplementary Table 3 are given as mean \pm s.d. b, $K_{\mathrm{M}} / V_{\max }$ determination for the first, second and third CK1 phosphorylation steps. The $v_{\max }$ for the phosphorylation of S588 is reduced by a factor of $\sim 2$ compared with $S 585$. The corresponding $K_{\mathrm{M}}$ drops by a factor of $\sim 7$. The $K_{M}$ and $v_{\max }$ values of $\mathrm{S} 591$ could not be determined due to the need for exceedingly high peptide concentrations. $K_{\mathrm{M}}$ and $v_{\max }$ determination was performed in triplicates for S585 and S588 phosphorylation and as a duplicate for $\mathbf{5} 591$ phosphorylation. The curves show one experiment out of these replicates. See Extended Data Fig. 5b and Supplementary Table 5. Values in Supplementary Table 5 are given as mean \pm s.d.

Consistent with the NMR data, T594 phosphorylation is significantly slower in the protein as compared to S591, while phosphorylation of T594 occurs almost as fast as S591 in the peptide. This suggests that T594 phosphorylation within the protein is sterically hindered due to its proximity to the inhibitory $\beta$-sheet.

Structure of the CK1 p63-PAD complex. We hypothesized that the residues C-terminal to the third CK1 phosphorylation site (S591) might be responsible for the observed kinetics. We exchanged the sequence following the third CK1 site S591 (S592 and E593) with the sequence directly C-terminal to S588 (V589 and G590). Interestingly, phosphorylation of S591 and T594 in this mutant was indeed 10-fold faster compared to the wild type. Faster phosphorylation kinetics were also evident in both single S592V and E593A mutants, with a more pronounced effect for the S592V mutation (Extended Data Fig. 2 and Supplementary Table 1).

To obtain more mechanistic insight into the kinase-peptide interactions, we determined the crystal structures of the kinase domain of $\mathrm{CK} 1 \delta$ in complexes with $\beta, \gamma$-methyleneadenosine $5^{\prime}$-triphosphate (AMPPCP) / adenosine $5^{\prime}$-diphosphate (ADP) and PAD-1P (phosphorylated at pS582), PAD-2P (pS582 and pS585) and PAD-3P (pS582, pS585 and pS588) (Extended Data Fig. 3 and Supplementary Table 2). The overall conformations of all bound peptides were highly similar. However, they captured different states, including the substrate-bound form in PAD-1P and PAD-2P, having the S585 and S588, respectively, located at the catalytic site. In contrast to $\mathrm{PAD}-1 \mathrm{P} / 2 \mathrm{P}$, the $\mathrm{PAD}-3 \mathrm{P}$ complex crystalized in a product-bound state in which pS588 was positioned within the catalytic site (Fig. 4). Structural analyses of the CK18-PAD-2P complex revealed that the interactions were induced by the electrostatic charge complementarity of both anchor ends on the $\mathrm{N}$ and $\mathrm{C}$ termini of the peptide (Fig. 4b). The peptide N-terminal part adopted a helical turn, positioning the phosphate of pS582 and pS585 into the basic pocket of the kinase formed by Arg178 and Lys224 (for clear referencing, we use the single amino acid letter code for the PAD peptide and the three-letter code for the kinase), while the PAD C-terminal E593 residue interacted with a second basic cluster present in the CK1 $\delta$ substrate-binding groove. This second cluster was formed by the $\mathrm{N}$ terminus of the activation segment of the kinase. The middle segment of the PAD central to S588 at the catalytic site displayed a canonical kinase-substrate interaction (Fig. $4 c, d)$. Close inspection of the region surrounding the catalytic site revealed a nonpolar patch on the kinase that was compatible with a medium-sized hydrophobic substrate residue like the V589 of the PAD peptide. This explains the preference for hydrophobic amino acids in CK substrates at the +1 position relative to the phosphorylation site (Fig. 4c,d). By comparison, the binding mode of PAD-3P in a product-bound state greatly resembles that of the substrate PAD-2P, albeit with the positioning of pS588 at the catalytic site with its phosphate moiety adjacent to the DFG motif (Asp-Phe-Gly triad) Asp149 and the HRD motif (His-Arg-Asp triad) Asp128, facilitated by an unusually bent ADP conformation (Fig. 4c,d). These structural insights from both substrate- and product-bound states suggested that accommodation of the PAD peptides with S588/pS588 at the catalytic site probably offered an optimal binding mode within the kinase, presenting the charge compatibility not only at $\mathrm{N}$ - and C-terminal anchor points but also the nonpolar groove for the middle part. Processivity towards the next phosphorylation event on S591 requires the movement of the triple phosphorylated peptide by translocation of S592 and E593 away from the polar/charged pocket to the unfavorable hydrophobic environment, explaining the accelerated kinetics of the mutants (Extended Data Fig. 2). To further investigate this model, we measured the phosphorylation kinetics for the V589A mutant, replacing a large hydrophobic residue with a smaller one. This mutation indeed slowed phosphorylation of the second phosphorylation site approximately threefold, probably by weakening the interaction with the hydrophobic surface on CK1. At the same time, the V589A mutation accelerated phosphorylation of S591 approximately threefold (Extended Data Fig. 2 and Supplementary Table 1). Replacing the medium-sized hydrophobic valine with alanine probably weakened the interaction in this +1 substrate position, resulting in both suboptimal positioning of the substrate and a smaller hurdle for release of the product.

We also investigated the interaction between the kinase and the PAD peptide by all-atom molecular dynamics (MD) simulations. In 1- $\mu$ s-long simulations, the side chain of E593 of the PAD peptide formed strong and persistent salt-bridge interactions with a basic cluster on CK1 formed by Arg127, Lys154 and Lys171 (Fig. 5a,b, and Extended Data Fig. 4a,b). By stabilizing this position of the peptide, these interactions contribute to the slow phosphorylation rate 
of S591, consistent with the mutational analysis of the PAD peptide showing an approximately twofold acceleration for the E593A mutant (Extended Data Fig. 2 and Supplementary Table 1). Mutating the residues of the basic cluster on CK1 individually to glutamate revealed that mutation of Lys154 and Lys171 resulted in slightly faster kinetics of the third CK1 phosphorylation reaction (Extended Data Fig. 4c). These results confirmed the contribution of the interaction between E593 and the kinase to the slow phosphorylation rate of S591.

The MD simulations also highlighted the stabilizing interactions of V589 with CK1. We compared two 1- $\mu$ s-long simulations of the wild-type and V589A PAD peptide. The V589A mutation weakened the interactions with the hydrophobic patch on CK1 and increased the flexibility of the peptide (Fig. $5 c, d$ ). This finding is consistent with our mutational analysis, which showed that a large hydrophobic residue in the $i+1$ position retains the peptide and thereby slows down phosphorylation at the third CK1 site.

Given that all phosphorylation steps follow a sequential, distributive mechanism, the decrease in phosphorylation kinetics with higher phosphorylation levels might be due to a decrease in binding affinity of the increasingly phosphorylated peptides. Measuring the affinity of all three phosphopeptides to CK1 $\delta$ using fluorescence anisotropy showed that all bind with a similar micromolar affinity (Fig. 6a, Extended Data Fig. 5a and Supplementary Table 3). These measurements, however, only report the overall affinity of the peptides, which can bind in different ways (product- or substrate-bound) to the kinase. For further analysis we measured the enzyme kinetics for single phosphorylation events. We thus produced $\mathrm{PAD}-1 \mathrm{P}, \mathrm{PAD}-2 \mathrm{P}$ and $\mathrm{PAD}-3 \mathrm{P}$ peptides in which we restricted the possible phosphorylation events by serine to alanine mutations (Supplementary Table 4) and monitored the phosphorylation kinetics using MS. The results show that the maximum reaction rate $\left(v_{\max }\right)$ values decrease with higher phosphorylation levels. The $K_{\mathrm{M}}$ value first decreases but drastically increases for the PAD-3P peptide (Fig. 6b, Extended Data Fig. 5b and Supplementary Table 5). In combination with the $K_{\mathrm{D}}$ measurements and the MD simulations, these results suggest that the PAD-3P peptide prefers to bind in a product-type state, as seen in the crystal structure. Binding in the substrate-bound position as necessary for phosphorylation of S591 is less favorable, explaining the slow kinetics for S591.

\section{Discussion}

Many theoretical and experimental studies have shown how cells build switch-like systems, often using multisite phosphorylation as a signal integrator of the activities of two or more kinases. Although activation of TAp63 $\alpha$ requires two kinases, this integrator model does not apply here, because CK1 kinases are thought to be constitutively active but sequentially dependent on a priming kinase such as CHK2. Thus, activation of TAp63 $\alpha$ would only depend on the activation of CHK2. This model, however, is only valid if CK1 uses a strictly processive mode of phosphorylation with all individual kinetic constants in the same range. Our results show that both conditions are not true and that CK1 uses a sequential distributive mode in combination with very different kinetic constants to regulate TAp63 $\alpha$ 's activation. Importantly, it is the third phosphorylation that is the slowest and that constitutes the decisive phosphorylation for formation of the open, tetrameric state. Transition to the tetrameric state is irreversible, and all phosphates can be removed in this state without affecting its oligomerization status ${ }^{8}$. Phosphorylation of the third CK1 site constitutes the point of no return. Mechanistically, the slow kinetics seems to be the result of three effects: (1) S592 and E593 interact with a basic/polar cluster of the kinase; (2) hydrophobic residues such as valine bind preferably in the +1 position relative to the phosphorylation site due to the small hydrophobic patch next to the active site (shifting S592 into this position is unfavorable); (3) the triple phosphorylated peptide (pS582, pS585, pS588) has a high affinity to the kinase in a product-type state. The crystal structure of the PAD-3P with pS588 pointing into the active site suggested product inhibition might play a role in slowing down phosphorylation of S591. The high $K_{\mathrm{M}}$ value for the PAD-3P peptide, combined with the $K_{\mathrm{D}}$ value's insensitivity towards phosphorylation, further supports the interpretation that binding in the product state is favorable relative to the substrate-bound state. Our findings agree with a study showing that temperature compensation of the circadian clock, in which CK1 is a central component, is partially based on a shift from high substrate affinity to higher product affinity ${ }^{39}$.

Sequence alignments of the $\mathrm{p} 63-\mathrm{PAD}$ peptide revealed that the phosphorylation sites and also the valine-glycine sequence following the second CK1 site and the serine-glutamate sequence C-terminal to the third site are highly conserved from fish to mammals ${ }^{12}$. Activation of TAp63 $\alpha$ could follow a universal, evolutionarily conserved mechanism with similar activation kinetics. Analysis of the phosphorylation kinetics and sequence of the YAP peptide supports this interpretation. This peptide does not show strong differences between all three phosphorylation events. The second and third phosphorylation sites have a large hydrophobic amino acid in the $n+1$ position (methionine and tyrosine, respectively), which interact favorably with the hydrophobic patch. Interestingly, the first site has a glycine following the serine. This amino acid does not provide optimal positioning of the peptide for modification, as shown by the MD simulation. Phosphorylation of this first site is much slower than the first two sites of the p63-PAD sequence, resulting in overall slower, but more similar kinetic constants for the three sites of the YAP sequence.

Importantly, the general behavior of the individual phosphorylation events in TAp63 $\alpha$ is not restricted to the relatively artificial phosphorylation of isolated peptides. It can also be seen in the kinetic analysis of the same sequence within the full-length TAp $63 \alpha$ protein. Measurements with full-length TAp63 $\alpha$ suggested that phosphorylation of S591 is faster than in the isolated peptides. As mentioned above, this might be a consequence of the dimeric nature of TAp63 $\alpha$ containing two kinase binding sites within one molecule and potentially other interaction sites that increase the local concentration of the kinase. The slower diffusion rate of TAp63 $\alpha$ relative to the peptide might also contribute to this effect by increasing the local concentration and making the kinase rebinding more efficient. However, T594 phosphorylation is slower, consistent with its position close to the start of the $\beta$-strand of the TID ${ }^{12,16}$. The $\beta$-strand is part of the six-stranded anti-parallel $\beta$-sheet that keeps TAp63 $\alpha$ in its dimeric state. Steric hindrance is expected for the T594 modification, making its phosphorylation slower in TAp63 $\alpha$ than in the isolated peptide.

Beyond the mechanistic description of the activation process, the question of the biological purpose of the observed biphasic kinetics remains. Delaying of the irreversible spring-loaded activation by the slow third phosphorylation might allow the cell to survive in cases where the DNA damage does not surpass a certain threshold. Phosphatases could act during the delay period and remove the phosphorylation sites that constitute the buffer. One additional safety mechanism is the degradation of activated TAp63 $\alpha$. Indeed, analysis of the total TAp $63 \alpha$ concentration in oocytes has revealed that it decreases following activation to the active tetrameric state ${ }^{3,8}$. A fast degradation in combination with slow activation could prevent apoptosis when the DNA damage level is minor. In such a model, the fast and slow kinetics ratio would set the threshold of DNA damage that triggers apoptosis. This threshold level has been established during evolution to suppress oocyte loss due to accidental activation of TAp $63 \alpha$ or low levels of DNA damage caused by background radioactivity or reactive oxygen species in oocytes.

\section{Online content}

Any Nature Research reporting summaries, source data, extended data, supplementary information, acknowledgements, peer review information; details of author contributions and competing interests; and statements of data and code availability are available at https://doi.org/10.1038/s41589-020-0600-3. 
Received: 26 June 2019; Accepted: 25 June 2020;

Published online: 27 July 2020

\section{References}

1. Johnston, R. J. \& Wallace, W. H. Normal ovarian function and assessment of ovarian reserve in the survivor of childhood cancer. Pediatr. Blood Cancer 53, 296-302 (2009).

2. Maltaris, T., Beckmann, M. W. \& Dittrich, R. Review. Fertility preservation for young female cancer patients. Vivo 23, 123-130 (2009).

3. Suh, E. K. et al. p63 protects the female germ line during meiotic arrest. Nature 444, 624-628 (2006).

4. Wallace, W. H., Thomson, A. B. \& Kelsey, T. W. The radiosensitivity of the human oocyte. Hum. Reprod. 18, 117-121 (2003).

5. Quast, U. Whole body radiotherapy: a TBI-guideline. J. Med. Phys. 31, 5-12 (2006).

6. Woodard, T. L. \& Bolcun-Filas, E. Prolonging reproductive life after cancer: the need for fertoprotective therapies. Trends Cancer 2, 222-233 (2016).

7. Livera, G. et al. p63 null mutation protects mouse oocytes from radio-induced apoptosis. Reproduction 135, 3-12 (2008).

8. Deutsch, G. B. et al. DNA damage in oocytes induces a switch of the quality control factor TAp63 $\alpha$ from dimer to tetramer. Cell 144, 566-576 (2011)

9. Kerr, J. B. et al. DNA damage-induced primordial follicle oocyte apoptosis and loss of fertility require TAp63-mediated induction of Puma and Noxa. Mol. Cell 48, 343-352 (2012).

10. Kim, S. Y. et al. Transient inhibition of $\mathrm{p} 53$ homologs protects ovarian function from two distinct apoptotic pathways triggered by anticancer therapies. Cell Death Differ. 26, 502-515 (2019).

11. Bolcun-Filas, E., Rinaldi, V. D., White, M. E. \& Schimenti, J. C. Reversal of female infertility by Chk2 ablation reveals the oocyte DNA damage checkpoint pathway. Science 343, 533-536 (2014).

12. Tuppi, M. et al. Oocyte DNA damage quality control requires consecutive interplay of CHK2 and CK1 to activate p63. Nat. Struct. Mol. Biol. 25 261-269 (2018).

13. Cesaro, L. \& Pinna, L. A. The generation of phosphoserine stretches i n phosphoproteins: mechanism and significance. Mol. Biosyst. 11, 2666-2679 (2015)

14. Knippschild, U. et al. The CK1 family: contribution to cellular stress response and its role in carcinogenesis. Front Oncol. 4, 96 (2014).

15. Schittek, B. \& Sinnberg, T. Biological functions of casein kinase 1 isoforms and putative roles in tumorigenesis. Mol. Cancer 13, 231 (2014).

16. Coutandin, D. et al. Quality control in oocytes by p63 is based on a spring-loaded activation mechanism on the molecular and cellular level. eLife 5, e13909 (2016)

17. Prehoda, K. E., Scott, J. A., Mullins, R. D. \& Lim, W. A. Integration of multiple signals through cooperative regulation of the N-WASP-Arp2/3 complex. Science 290, 801-806 (2000).

18. Chen, L., Glover, J. N., Hogan, P. G., Rao, A. \& Harrison, S. C. Structure of the DNA-binding domains from NFAT, Fos and Jun bound specifically to DNA. Nature 392, 42-48 (1998).

19. Rinaldi, V. D., Hsieh, K., Munroe, R., Bolcun-Filas, E. M. \& Schimenti, J. C. Pharmacological inhibition of the DNA damage checkpoint prevents radiation-induced oocyte death. Genetics 206, 1823-1828 (2017).
20. Carmell, M. A. et al. A widely employed germ cell marker is an ancient disordered protein with reproductive functions in diverse eukaryotes. eLife $\mathbf{5}$, e19993 (2016)

21. Keller, P. J., Schmidt, A. D., Wittbrodt, J. \& Stelzer, E. H. Digital scanned laser light-sheet fluorescence microscopy (DSLM) of zebrafish and Drosophila embryonic development. Cold Spring Harb. Protoc. 2011, 1235-1243 (2011).

22. Susaki, E. A. et al. Whole-brain imaging with single-cell resolution using chemical cocktails and computational analysis. Cell 157, 726-739 (2014).

23. Hotte, K. et al. Ultra-thin fluorocarbon foils optimise multiscale imaging of three-dimensional native and optically cleared specimens. Sci. Rep. 9, 17292 (2019).

24. Ferrell, J. E. Jr \& Ha, S. H. Ultrasensitivity part III: cascades, bistable switches and oscillators. Trends Biochem. Sci. 39, 612-618 (2014).

25. Ferrell, J. E. Jr \& Ha, S. H. Ultrasensitivity part II: multisite phosphorylation, stoichiometric inhibitors and positive feedback. Trends Biochem. Sci. 39, 556-569 (2014)

26. Ferrell, J. E. Jr \& Ha, S. H. Ultrasensitivity part I: Michaelian responses and zero-order ultrasensitivity. Trends Biochem. Sci. 39, 496-503 (2014).

27. Ferrell, J. E. Jr \& Bhatt, R. R. Mechanistic studies of the dual phosphorylation of mitogen-activated protein kinase. J. Biol. Chem. 272, 19008-19016 (1997).

28. Serber, Z. et al. A C-terminal inhibitory domain controls the activity of p63 by an intramolecular mechanism. Mol. Cell Biol. 22, 8601-8611 (2002).

29. Straub, W. E. et al. The C-terminus of p63 contains multiple regulatory elements with different functions. Cell Death Dis. 1, e5 (2010).

30. Selenko, P. et al. In situ observation of protein phosphorylation by high-resolution NMR spectroscopy. Nat. Struct. Mol. Biol. 15, 321-329 (2008).

31. Cordier, F. et al. Ordered phosphorylation events in two independent cascades of the PTEN C-tail revealed by NMR. J. Am. Chem. Soc. 134, 20533-20543 (2012)

32. Narasimamurthy, R. et al. CK1 $\Delta \varepsilon$ protein kinase primes the PER2 circadian phosphoswitch. Proc. Natl Acad. Sci. USA 115, 5986-5991 (2018).

33. Mylona, A. et al. Opposing effects of Elk-1 multisite phosphorylation shape its response to ERK activation. Science 354, 233-237 (2016).

34. Leroy, A. et al. Spectroscopic studies of GSK3 $\beta$ phosphorylation of the neuronal Tau protein and its interaction with the N-terminal domain of apolipoprotein E. J. Biol. Chem. 285, 33435-33444 (2010).

35. Philpott, J. M. Casein kinase 1 dynamics underlie substrate selectivity and the PER2 circadian phosphoswitch. eLife 9, e52343 (2020).

36. Theillet, F. X. et al. Sensitivity-enhanced ${ }^{13} \mathrm{C}-\mathrm{NMR}$ for monitoring multisite phosphorylation at physiological temperature and $\mathrm{pH}$. Angew. Chem. Int. Ed. 59, 10411-10415 (2020).

37. Favier, A. \& Brutscher, B. Recovering lost magnetization: polarization enhancement in biomolecular NMR. J. Biomol. NMR 49, 9-15 (2011).

38. Zhao, B., Li, L., Tumaneng, K., Wang, C. Y. \& Guan, K. L. A coordinated phosphorylation by Lats and CK1 regulates YAP stability through SCF( $\beta$-TRCP). Genes Dev. 24, 72-85 (2010).

39. Shinohara, Y. et al. Temperature-sensitive substrate and product binding underlie temperature-compensated phosphorylation in the clock. Mol. Cell 67, 783-798 (2017).

Publisher's note Springer Nature remains neutral with regard to jurisdictional claims in published maps and institutional affiliations.

(c) The Author(s), under exclusive licence to Springer Nature America, Inc. 2020 


\section{Methods}

Ovary culture. Animal care and handling were performed according to World Health Organization (Geneva, Switzerland) guidelines. Eight-day-old (P8) female CD-1 mice were purchased from Charles River Laboratories. Ovaries were harvested, transferred to sterile 96-well plates with $50 \mu \mathrm{l} \alpha$-MEM (+L-Glu, Gibco) supplemented with $10 \%$ FBS (Gibco), $1 \times$ penicillin/streptomycin (Gibco), $0.2 \mathrm{mg} \mathrm{ml}^{-1} \mathrm{Na}$-pyruvate (Gibco), $2 \mathrm{mg} \mathrm{ml}^{-1} \mathrm{~N}$-acetyl-L-cysteine (Sigma) and ITS liquid medium supplement (100X; Sigma) cultured at $37^{\circ} \mathrm{C}$ with $5 \% \mathrm{CO}^{2}$ overnight ${ }^{40}$. The final concentrations of the kinase inhibitors targeting ATM (KU55399, Selleckchem), CHK2 (BML-277, Merck) and CK1 (PF670462, Sigma Aldrich) were $25 \mu \mathrm{M}$ or $50 \mu \mathrm{M}$. Ex vivo ovaries were gamma-irradiated with $0.50 \mathrm{~Gy}$ on a rotating turntable in a ${ }^{137} \mathrm{Cs}$ irradiator at a dose of $2.387 \mathrm{~Gy} \mathrm{~min}^{-1}$. The Tierschutzbeauftragte of the Goethe University Frankfurt am Main approved the protocol for harvesting mouse ovaries.

Western blotting and blue native polyacrylamide gel electrophoresis. For each time point, four ovaries were used. After the indicated time following irradiation, lysates were produced, and western blotting and BN-PAGE were performed as described previously ${ }^{12,29}$ with the same lysate always analyzed both by SDS-PAGE and BN-PAGE. The following antibodies were used for detection: anti-p63 $\alpha$ (D2K8K XP, Cell Signaling), anti-cleaved-PARP (D6X6×, Cell Signaling), anti-VASA (DDX-4) (ab13840, Abcam) and anti-Msy2 (N-13, SCBT). The densitometry analysis was performed using Fiji. Each lane was integrated, dimer and tetramer fractions were gated, and the percentage of each gate was calculated in relation to the total integral of the lane.

\section{Whole ovary staining, optical clearing and light sheet-based fluorescence} microscopy. Cultured ovaries were treated as indicated, and the staining was performed as described in ref. ${ }^{12}$. In brief, the ovaries were harvested and fixed in $4 \%$ paraformaldehyde (PFA) in PBS overnight at $4{ }^{\circ} \mathrm{C}$. The ovaries were permeabilized using $0.3 \%$ Triton X-100 in PBS for $30 \mathrm{~min}$ at room temperature. All further steps were performed in a 96-well flat-bottom plate (Greiner) shaking at 450 r.p.m. The ovaries were blocked for $2 \mathrm{~h}$ using blocking buffer $(0.3 \%$ Triton $\mathrm{X}-100,0.05 \%$ Tween-20, $0.1 \%$ BSA (heat shock fraction, Sigma) and $10 \%$ donkey serum in PBS), then the ovaries were incubated overnight at $37^{\circ} \mathrm{C}$ in a humidified incubator with the first antibody, an anti-germ cell-specific antigen (GCNA1; ab82527, Abcam) 1:200 and anti-cleaved PARP (D6X6X, Cell Signaling) and $1 \mu \mathrm{g} \mathrm{ml}^{-1}$ DAPI, which was diluted in blocking buffer. This was followed by three 20-min PBS washes and a subsequent 4-h incubation of the secondary antibody, donkey anti-rabbit Alexa 488 (A-21206, Thermo Fisher) and donkey anti-rat Alexa 568 (ab1754775, Abcam), in a 1:200 dilution in blocking buffer containing $1 \mu \mathrm{g} \mathrm{ml} l^{-1} \mathrm{DAPI}$ at $37^{\circ} \mathrm{C}$ in a humidified incubator in the dark. The ovaries were washed three times for $20 \mathrm{~min}$ with PBS and kept at $4{ }^{\circ} \mathrm{C}$ in PBS. The ovaries were cleared by washing them four times in CUBIC $2^{22}(50 \% \mathrm{wt} / \mathrm{vol}$ sucrose, $25 \% \mathrm{wt} /$ vol urea, $10 \% \mathrm{wt} / \mathrm{vol} 2,2^{\prime}, 2^{\prime \prime}$-nitrilotriethanol) with a refraction index of 1.49 and overnight incubation in CUBIC 2 inside a fluorinated ethylene propylene (FEP) foil capillary ${ }^{23}$ (US patent US $20150211981 \mathrm{Al}^{41}$ ). The capillaries were mounted on stainless steel holders, and images were acquired with a custom-built monolithic digital-scanned light sheet-based fluorescence microscope (mDSLM) ${ }^{21}$ The microscope was equipped with an Epiplan-Neofluar $\times 2.5 / 0.06$ illumination objective and an N-Achroplan $\times 10 / 0.3$ detection objective (Carl Zeiss) and a Clara camera (ANDOR Technology) ( $z$-spacing of $2.58 \mu \mathrm{m}$; laser and filter set: 488-nm laser, 525/50 bandpass filter). All raw image stacks were pre-processed in Fiji (ImageJ version 1.51d, Java version 1.6.0_24). Specifically, the raw image stacks were cropped to the region of interest and scaled by a factor of two. Homogeneous intensity was obtained by distributing the individual images of the $z$-stacks, and the stacks were re-sliced by a factor of four. The background intensity was subtracted using the Fiji function Subtract Background with a ball radius of 50 pixels, and the contrast was enhanced by applying the Fiji function Enhance Contrast (saturation $=0.35$ ). GCNA and c-PARP positive cells were segmented with the Fiji function 3D Object Counter. Objects with min. $=750$ and max. $=222,218,880$ voxels and an intensity threshold of 1,000 were segmented. The total amounts of cPARP- and GCNA-positive cells per ovary were extracted, and the ratio of GCNA/c-PARP per ovary was calculated. Three replicates per time point were analyzed.

TAp63 $\alpha$ expression, purification and phosphorylation. Human TAp63 $\alpha$ (amino acids 10-616, TEV site inserted at amino acids 570 and V599M) or T586A mutant were codon-optimized for expression in Escherichia coli and subcloned into the pET16b vector. The protein, bearing a C-terminal $\mathrm{His}_{6}$-tag, was expressed in BL-21(DE3)-R3-Rosetta (SGC Oxford) for $16 \mathrm{~h}$ at $18^{\circ} \mathrm{C}$ in M9 minimal medium containing $1 \mathrm{gl}^{-1}{ }^{15} \mathrm{NH}_{4} \mathrm{Cl}$ as the nitrogen source. The medium was also supplemented with $100 \mu \mathrm{M} \mathrm{ZnCl}_{2}$ to ensure correct folding of the zinc finger containing the DNA binding domain. Cells were lysed in IMAC buffer A (50 mM Tris pH 8.0, $400 \mathrm{mM} \mathrm{NaCl}, 20 \mathrm{mM} \beta$-mercaptoethanol, $5 \%$ glycerol, $10 \mu \mathrm{M} \mathrm{ZnCl}_{2}$ ) and purified using a standard step gradient $(300 \mathrm{mM}$ imidazole) immobilized metal affinity chromatography (IMAC) protocol (Ni-Sepharose Fast Flow, GE Healthcare). Next, the protein was further purified by size exclusion chromatography (SEC; HiLoad 16/600 Superdex 200 pg, GE Healthcare) in
$50 \mathrm{mM}$ Tris pH 8.0, $150 \mathrm{mM} \mathrm{NaCl}, 5 \%$ glycerol, $0.5 \mathrm{mM}$ TCEP. Purified TAp63 $\alpha$ was incubated for $30 \mathrm{~min}$ at $30^{\circ} \mathrm{C}$ with pre-activated MK2 $\Delta 1-41,10 \mathrm{mM}$ ATP and $10 \mathrm{mM} \mathrm{MgCl}_{2}$. Purification and activation of MK2 $\Delta 1-41$ were performed as described previously ${ }^{12}$. Subsequently, TAp63 $\alpha$ was separated from MK2 by SEC (HiLoad 16/600 Superdex $200 \mathrm{pg}$, GE Healthcare) in $50 \mathrm{mM}$ Tris pH 8.0, $150 \mathrm{mM}$ $\mathrm{NaCl}, 5 \%$ glycerol and $0.5 \mathrm{mM}$ TCEP, then $2.5 \mathrm{mg}$ of the pre-phosphorylated TAp63 $\alpha$ (pTAp63 $\alpha$ ) was incubated with purified CK1 $\delta$ (molar ratio 1:1,000 or 1:100) with $10 \mathrm{mM}$ ATP and $10 \mathrm{mM} \mathrm{MgCl}_{2}$ at $25^{\circ} \mathrm{C}$ for the indicated time. Purification of CK1 $\delta$ was performed as described previously ${ }^{12}$. To stop the reaction, PF670462 $(10 \mu \mathrm{M}$ final concentration) and EDTA pH $8.0(12 \mathrm{mM}$ final concentration) were added. MBP-TEV was then added in a 1:1 molar ratio and incubated overnight at $4^{\circ} \mathrm{C}$. Cleavage was stopped by adding solid urea to a final concentration of $6 \mathrm{M}$. The denaturated mixture was concentrated and purified by SEC (Superdex 75, 10/300 GL, GE Healthcare) in $50 \mathrm{mM}$ Tris pH 8.0, $150 \mathrm{mM}$ $\mathrm{NaCl}$ and $6 \mathrm{M}$ urea. The resulting PAD-TID peptide was acidified with $32 \% \mathrm{HCl}$ to a final concentration of $0.5 \mathrm{M}$ and cleaved by a final concentration of $125 \mathrm{mM}$ $\mathrm{CNBr}$ for $48 \mathrm{~h}$ at room temperature in the dark. The resulting product was concentrated for $30 \mathrm{~min}$ in a SpeedVac. Afterward, MES pH 6.3 was added to a final concentration of $500 \mathrm{mM}$ to adjust the $\mathrm{pH}$.

A reverse IMAC removed the TID. The sample was concentrated and buffer exchanged (kinase NMR buffer, see below) by ultrafiltration (Amicon Ultra $0.5 \mathrm{ml}, 3-\mathrm{kDa}$ molecular weight cutoff (MWCO), Merck) for NMR or MS analysis. T586A mutant was used for the MS analysis to be able to distinguish between the phosphorylated states.

Peptide expression. Peptides were expressed with a GFP leader protein to increase the protein expression yield in E. coli (architecture of the construct: GFP-His ${ }_{6}-3$ Csite-peptide). Isotopically labeled expression was performed in M9 medium for $16 \mathrm{~h}$ at $22^{\circ} \mathrm{C}$ under induction with $500 \mu \mathrm{M}$ IPTG. Initial purification was performed analogous to full-length TAp63 $\alpha$ including IMAC purification (buffer A: $25 \mathrm{mM}$ Tris pH 8.0, $200 \mathrm{mM} \mathrm{NaCl}, 30 \mathrm{mM}$ imidazole; washing with five column volumes, elution with buffer B: $25 \mathrm{mM}$ Tris pH 8.0, $200 \mathrm{mM} \mathrm{NaCl}, 500 \mathrm{mM}$ imidazole). The expression tag and the peptide were cleaved by incubation with $3 \mathrm{C}$ protease overnight at $4^{\circ} \mathrm{C}$. The next day, the peptide and GFP were subject to concentration via ultrafiltration (Amicon Ultra 10-kDa MWCO, Merck) and to remove the GFP and $3 \mathrm{C}$ protease. The flowthrough of the filter was subjected to another round of concentration over a 3-kDa cutoff filter. Afterward, the peptides were subjected to SEC in kinase NMR buffer (Superdex 75 10/300 GL, GE Healthcare) (see below).

Different phospho-states of the peptides were generated by carrying out phosphorylation reactions and quenching them by the addition of EDTA to remove all available magnesium ions. Next, the peptide mix was subject to buffer exchange into anion exchange buffer A ( $25 \mathrm{mM}$ Tris $\mathrm{pH}$ 8.0) over a HiTrap desalting column (GE Healthcare). In the next step, the sample was bound to a HiTrap Q HP anion exchange chromatography column (GE Healthcare) and eluted with a linear gradient to anion exchange buffer B $(25 \mathrm{mM}$ Tris $\mathrm{pH} 8.0,500 \mathrm{mM}$ $\mathrm{NaCl}$ ) with a flow rate of $5 \mathrm{ml} \mathrm{min}^{-1}$ over a time of $20 \mathrm{~min}$. Peak fractions were collected, concentrated and subjected to MS to determine the phosphorylation state and purity of the peptide.

Peptide MK2 pre-phosphorylation. Peptides were S582-phosphorylated with MK2 kinase at $25^{\circ} \mathrm{C}$ at a molar ratio of 1:100 kinase to peptide in kinase NMR buffer supplemented with ATP at a concentration of $10 \mathrm{mM}$. Phosphorylation was monitored by recording a series of $2 \mathrm{D}$ NMR spectra. MK2 was subsequently removed from the reaction mix by another round of SEC (Superdex 75, 10/300 GL, GE Healthcare) in kinase NMR buffer.

Nuclear magnetic resonance spectroscopy. Samples for NMR experiments were used in kinase NMR buffer (50 mM Bis-Tris pH 6.5, $50 \mathrm{mM} \mathrm{NaCl}, 10 \mathrm{mM}$ $\mathrm{MgCl}_{2}$ ). All samples contained $1 \times$ protease inhibitor cocktail (complete EDTA free, Roche) and $1 \times$ phosphatase inhibitor cocktail (PhosSTOP, Roche). Experiments were performed at a sample temperature of $298 \mathrm{~K}$. Assignments of PAD mutants at different phosphorylation states were performed using a combinatorial triple-selective labeling approach, as detailed in ref. ${ }^{42}$, or with the help of a constant-time HNCACB.

Phosphorylation kinetics were recorded using sample volumes of $200 \mu \mathrm{l}$ placed in 3-mm capillaries and final peptide concentrations of $250 \mu \mathrm{M}$. The kinase concentration was either $125 \mathrm{nM}(1: 2,000)$ or $12.5 \mathrm{nM}(1: 20,000)$. All NMR experiments with peptides were performed at least twice under identical conditions. The general sample composition of a kinetic sample was as follows: ${ }^{15} \mathrm{~N}$-labeled peptide $(250 \mu \mathrm{M})$, protease inhibitor $(1 \times)$, phosphatase inhibitor $(1 \times)$, ATP $(10 \mathrm{mM})$, CK1 kinase ( $125 \mathrm{nM}$ or $12.5 \mathrm{nM}$ ) and buffer (to $200 \mu \mathrm{l}$ ).

A series of HSQC-like $\left[{ }^{15} \mathrm{~N},{ }^{1} \mathrm{H}\right]$ correlation spectra were recorded throughout the kinetic reaction with NMR instruments operating at ${ }^{1} \mathrm{H}$ frequencies ranging from 600 to $950 \mathrm{MHz}$. Kinetics for the PAD peptides were measured by $600 \mathrm{MHz}$ or $700 \mathrm{MHz}$ spectrometers, the kinetics for the YAP peptide at $800 \mathrm{MHz}$, and the kinetics with the peptide:kinase ratio of 20,000:1 at $950 \mathrm{MHz}$.To accurately reflect the extremely fast initial phosphorylation reactions of S582 and S585, each spectrum's total measurement time was limited to a maximum of $120 \mathrm{~s}$. This 
was achieved using a gradient-selected BEST-TROSY pulse sequence ${ }^{37}$ and, by limiting the measurement to one scan per FID or by reducing the interscan delay to $100 \mathrm{~ms}$, depending on the field strength, the sample was measured. Non-uniform sampling was not employed, as the large quantity of ATP within the sample leads to non-recoverable $t_{1}$-noise-like artefacts, overlaying the signals.

Before the addition of kinase, a reference spectrum was acquired. Kinase was added manually and a series of spectra were recorded over a time course of $\sim 12 \mathrm{~h}$. Peak intensities of relevant peaks were quantified for every spectrum automatically. The fraction of phosphorylation in a given amino acid was calculated by dividing the relevant peak intensity/intensities by the sum of all peak intensities of a given amino acid.

YAP1 phosphorylation kinetics were measured based on ${ }^{13} \mathrm{C}$ detected $2 \mathrm{D}(\mathrm{H})$ NCO experiments to resolve the peak overlap in the $\left[{ }^{15} \mathrm{~N},{ }^{1} \mathrm{H}\right]-\mathrm{HSQC}$ spectrum. $2 \mathrm{D}{ }^{13} \mathrm{C}$ detected $\mathrm{N}-\mathrm{CO}$ correlation spectra were obtained using a BEST-(H)NCO pulse sequence, essentially as described in ref. ${ }^{43}$. Nitrogen chemical shifts were acquired in a semi-constant time manner using States-TPPI quadrature detection. The in-phase anti-phase (IPAP) approach achieved homonuclear ${ }^{13} \mathrm{CO}-{ }^{13} \mathrm{C} \alpha$ decoupling ${ }^{44}$. To allow for very short interscan delays without causing sample and RF coil heating, ${ }^{15} \mathrm{~N}$ decoupling during acquisition was not applied in the current implementation. The acquisition was started immediately after the 9.4-ms IPAP delay $\left(=\left(2^{1} J_{\mathrm{C} \alpha \mathrm{CO}}\right)^{-1}\right)$, leading to a slightly shorter pulse sequence duration. Refocusing of the active ${ }^{1} J_{\mathrm{CON}}$ coupling occurs during the fixed ${ }^{13} \mathrm{CO}-{ }^{13} \mathrm{C} \alpha$ IPAP delay and proceeds during acquisition. As a consequence, line shapes along the directly detected ${ }^{13} \mathrm{CO}$ dimension are a superposition of non-resolved in-phase ${ }^{1} J_{\mathrm{CON}}$ doublets, phased to absorption, and dispersive antiphase ${ }^{1} J_{\mathrm{CON}}$ doublets.

Spectra were recorded on a Bruker AVIII $800 \mathrm{MHz}$ spectrometer equipped with a cryogenic ${ }^{1} \mathrm{H} /{ }^{13} \mathrm{C} /{ }^{15} \mathrm{~N}$ triple-resonance TXO probe optimized for ${ }^{13} \mathrm{C}$ detection. Acquisition times were $60 \mathrm{~ms}$ for ${ }^{13} \mathrm{C}$ and $98.7 \mathrm{~ms}$ for ${ }^{15} \mathrm{~N}$ with spectral widths of $20 \mathrm{ppm}$ in both dimensions. Four FIDs were acquired for each time-domain data point in the indirect dimension (two for ${ }^{15} \mathrm{~N}$ quadrature detection and two for ${ }^{13} \mathrm{CO}-{ }^{13} \mathrm{C} \alpha$ IPAP), and two scans were accumulated for each FID. Using a relaxation delay of $0.1 \mathrm{~s}$ and non-uniform sampling ( $37.5 \%$ sparse), the total experimental time for each spectrum was $2 \mathrm{~min}$.

Phosphorylated Ser/Thr residues were identified based on ${ }^{3} J_{\mathrm{C} \alpha \mathrm{P}}$ couplings using a ${ }^{31} \mathrm{P}$-edited intra-HNCA experiment, as previously proposed ${ }^{45}$. To enable a direct comparison with the $\mathrm{N}-\mathrm{CO}$ correlation spectra acquired to monitor the phosphorylation reaction, a carbonyl evolution time $\left(t_{1}\right)$ was introduced here, resulting in the $3 \mathrm{D}\left[{ }^{15} \mathrm{~N},{ }^{1} \mathrm{H}\right]$-BEST-TROSY-COintraHN(CAP) pulse sequence shown in Supplementary Fig. 3e. Projection along the ${ }^{1} \mathrm{H}$ dimension of the final 3D spectrum provides the desired ${ }^{15} \mathrm{~N}_{i}-{ }^{13} \mathrm{CO}_{i-1}$ cross-peaks of phosphorylated residues $i$ (Supplementary Fig. 3c, green overlay in right panel).

The spectrum was acquired with a cryogenic ${ }^{1} \mathrm{H} /{ }^{31} \mathrm{P} /{ }^{13} \mathrm{C} /{ }^{15} \mathrm{~N}$ quadruple resonance QCI probe on a Bruker AVIIIHD 700- $\mathrm{MHz}$ spectrometer. Spectral widths were adjusted to 8,10 and $9.6 \mathrm{ppm}$, respectively, along the ${ }^{13} \mathrm{C},{ }^{15} \mathrm{~N}$ and ${ }^{1} \mathrm{H}$ dimensions, where the ${ }^{1} \mathrm{H}$ carrier was placed on the water frequency. Acquisition times were $53.8 \mathrm{~ms}\left({ }^{13} \mathrm{C}, 76\right.$ complex points $), 115.5 \mathrm{~ms}\left({ }^{15} \mathrm{~N}, 82\right.$ complex points) and $76.1 \mathrm{~ms}\left({ }^{1} \mathrm{H}, 512\right.$ complex points). Non-uniform sampling was employed to record a total of 2,181 hypercomplex points ( $35 \%$ of the full $t_{1} / t_{2}$ grid). The spectrum was acquired within $24 \mathrm{~h}$ using a recycle delay of $0.25 \mathrm{~s}$ and 16 scans per FID.

Nucler magnetic resonance kinetic analysis. The phosphorylation kinetics was analyzed according to a sequential model: $[\mathrm{A}] \rightarrow[\mathrm{B}] \rightarrow[\mathrm{C}] \rightarrow[\mathrm{D}]$, with [A] representing the CHK2-phosphorylated PAD peptide and [B], [C] and [D] representing successive phosphorylated species. The functions for fitting the experimental data were

$$
\begin{aligned}
& {[A]=[A]_{0} \mathrm{e}^{-k_{1} t}} \\
& {[B]=\frac{k_{1}[A]_{0}}{k_{2}-k_{1}}\left[\mathrm{e}^{-k_{1} t}-\mathrm{e}^{-k_{2} t}\right]} \\
& {[C]=k_{1} k_{2}[A]_{0}\left[\frac{1}{\left(k_{2}-k_{1}\right)\left(k_{3}-k_{1}\right)} \mathrm{e}^{-k_{1} t}-\frac{1}{\left(k_{2}-k_{1}\right)\left(k_{3}-k_{2}\right)} \mathrm{e}^{-k_{2} t}+\frac{1}{\left(k_{3}-k_{2}\right)\left(k_{3}-k_{1}\right)} \mathrm{e}^{-k_{3} t}\right]} \\
& {[D]=\frac{k_{2} k_{3}[A]_{0}}{\left(k_{2}-k_{1}\right)\left(k_{3}-k_{1}\right)}\left[1-\mathrm{e}^{-k_{1} t}\right]-\frac{k_{1} k_{3}[A]_{0}}{\left(k_{2}-k_{1}\right)\left(k_{3}-k_{2}\right)}\left[1-\mathrm{e}^{-k_{2} t}\right]-\frac{k_{1} k_{2}[A]_{0}}{\left(k_{3}-k_{1}\right)\left(k_{3}-k_{2}\right)}\left[1-\mathrm{e}^{-k_{3} t}\right]}
\end{aligned}
$$

Fitting and visualization were performed with the software package Prism 6.0 (GraphPad).

Crystal structure determination. The kinase domain of CK1 $1 \delta$ (amino acids 1-294) was subcloned into $\mathrm{pNIC} 28$-Bsa4, and the recombinant protein containing an $\mathrm{N}$-terminal $\mathrm{His}_{6}$ tag was expressed in E. coli, cultured in Terrific Broth (TB) and induced with $0.5 \mathrm{mM}$ IPTG at $18^{\circ} \mathrm{C}$ overnight. Cells were collected and lysed by sonication, and the protein was purified by IMAC. The histidine tag was cleaved by TEV protease treatment, and the cleaved protein was purified further by reverse IMAC and SEC. The pure protein in $20 \mathrm{mM}$ Tris, $\mathrm{pH} 7.5,200 \mathrm{mM} \mathrm{NaCl}$ and $0.5 \mathrm{mM}$ TCEP was concentrated to $6-8 \mathrm{mg} \mathrm{ml}^{-1}$ and mixed with either ADP or AMPPCP (Sigma) at $2 \mathrm{mM}$ and $\mathrm{MgCl}_{2}$ at $4 \mathrm{mM}$. Crystallization was performed using the sitting drop vapor diffusion method at $4{ }^{\circ} \mathrm{C}$ using the condition containing $10-20 \%$ PEG 3350, 0.1-0.2 M sodium sulfate and $0.1 \mathrm{M}$ citrate, $\mathrm{pH} 4.6-$ 5.9. Viable crystals were soaked with the PAD peptides at $6-10 \mathrm{mM}$ overnight in the mother liquor containing $20 \%$ ethylene glycol before being flash-cooled in liquid nitrogen. Diffraction data were collected at the Swiss Light Source, X06SA or DESY, P13, and were processed and scaled with $\mathrm{XDS}^{46}$ and subsequently scaled using AIMLESS ${ }^{47}$, respectively. The CK1 $\delta$ structures in complexes with the peptides were solved by molecular replacement using $\mathrm{Phaser}^{48}$ and the published coordinates of $\mathrm{CK} 1 \delta^{49}$. Manual model rebuilding alternating with structure refinement was performed in $\mathrm{COOT}^{50}$ and REFMAC ${ }^{51}$, respectively. Geometry correctness of the final structures was checked using MolProbity. Data collection and refinement statistics are summarized in Supplementary Table 2.

The peptides used for crystallization in complex with CK1 $\delta$ were as follows:

PAD-1P $=$ YTP $(\mathrm{pS})$ SASTVSVGSSET $\left(M_{\mathrm{w}}=1,639.6, e=1,490\right)$

PAD-2P $=$ YTP $(\mathrm{pS})$ SA $(\mathrm{pS})$ TVSVGSSET $\left(M_{w}=1,719.6, e=1,490\right)$

$\mathrm{PAD}-3 \mathrm{P}=(\mathrm{pS}) \mathrm{SA}(\mathrm{pS}) \mathrm{TV}(\mathrm{pS}) \mathrm{VGSSY}\left(M_{\mathrm{w}}=1,371.16, e=1,490\right)$ Facility.

The peptides were synthesized by the Tufts University Peptide Synthesis Core

Molecular dynamics simulations. Initial simulation models were built according to the X-ray crystal structure p63-PAD-3P, with bound ADP replaced by ATP. All crystallographic water molecules and ions within $10 \AA$ of the protein were retained. ATP and a complexed $\mathrm{Mg}^{2+}$ ion were added using Protein Data Bank entry 1CSN as a template, by superimposing the protein backbones and aligning the nitrogen atoms of ATP with the crystallographic ADP. Missing side chains were added using the software Modeller ${ }^{52}$. The triple phosphorylated peptide PAD-3P was elongated to TPpSSApSTVpSVGSSETRGER with charged termini, as used in the kinetic measurements using VMD Molefracture ${ }^{53}$ and Modeller (S592 and E593 coordinates from p63-PAD-2P). Using these tools, we also constructed a complex of CK1 with a shortened peptide ACE-TPpSSApSTVpSVGSSETRG-NME capped with $\mathrm{N}$-terminal acetyl and C-terminal methylamino capping groups. This peptide mimics the full-length protein, because E597 and R598 may not be accessible to CK1. In a third set-up, the point mutation V589A was introduced into the longer peptide using Modeller. In all three set-ups, Asp128 was protonated. All other residues were simulated in their physiological protonation state. All three MD simulations were carried out with GROMACS $2018^{54}$ using the AMBER99SB $^{*}$-ILDN-q force field ${ }^{55,56}$, the water model TIP3P and the Schwierz ion force field ${ }^{57}$ for $\mathrm{Mg}^{2+}$ and $\mathrm{NaCl}$ at $150 \mathrm{mM}$ concentration. Force-field parameters for ATP and phosphoserine were taken from ref. ${ }^{58}$ and ref. ${ }^{59}$, respectively. The system was energy minimized, followed by five equilibration steps with successively decreasing position restraints on heavy atoms, first in an NVT ensemble $(0.25 \mathrm{~ns})$ and then in an NPT ensemble $(4 \times 0.5 \mathrm{~ns})$ using a Berendsen thermostat and barostat. The three production runs of $1 \mu$ s each were run at a temperature of $310 \mathrm{~K}$ in an NPT ensemble using a Nosé-Hoover thermostat. The pressure was maintained at 1 bar with a Parrinello-Rahman barostat. The minimum heavy-atom distances between E593 and Arg127 and between E593 and Lys154, as well as the minimum heavy-atom distance between V589 or A589 (side chain) and the protein CK1 were monitored at 1-ns intervals using the gmx mindist tool. The raw distance data were processed using moving average smoothing with a window size of five. All structural figures were made using $\mathrm{VMD}^{53}$.

Matrix-assisted laser desorption/ionization mass spectrometry-based enzymatic assay. Previously published MALDI-MS-based peptide phosphorylation assays were adjusted and optimized for CK1 and the described peptides ${ }^{60,61}$. Briefly, purified kinase was diluted to a $50 \mathrm{nM}$ final assay concentration in the same buffer as described for the NMR experiments. Reactions were carried out with linear peptide dilutions and $4 \mathrm{mM}$ ATP at room temperature. Aliquots of these reactions were quenched by the addition of $0.1 \%$ TFA at certain time points, creating a time series of each peptide concentration. The stopped reactions were then spotted onto a target using a 4 -chloro- $\alpha$-cyanocinnamic acid matrix (Sigma Aldrich, $3 \mathrm{mg} \mathrm{ml}^{-1}$ in TFA-acidified $50 \%$ acetonitril). Each time point was spotted in quadruplets, measured on a Thermo Fisher MALDI-LTQ Orbitrap XL spectrometer, and analyzed with Xcalibur software (Thermo Fisher). The $m / z$ integrals over the measured intensities of the peptides were used to assess the relative phosphorylation state of the peptide (Supplementary Table 3). A factor $(F \mathrm{c})$ based on equation (1) was calculated for each peptide to correct for nonlinear ionization and ion mobility of the peptides with the higher phosphorylation state. An equimolar mixture of the same peptide with and without phosphorylation was measured, and $F \mathrm{c}$ was calculated. As validation of this factor, a linear series of increasing phosphorylated fraction of each peptide was obtained, and the relative intensity of the peptide with the higher phosphorylation state was calculated with and without Fc. Examples of these curves with improved linearity are shown in Extended Data Fig. 5c-e.

For the determination of $K_{\mathrm{M}}$ and $v_{\max }$ of each phosphorylation step, corrected, relative intensities of a given peptide concentration were plotted against the reaction time. The initial velocity was calculated by a linear fit function of Prism 8.1. These initial velocities were further analyzed as a function of the peptide concentration, and $K_{\mathrm{M}}$ and $v_{\max }$ values were calculated using a Michaelis-Menten fit of the Prism 8.1 software. The experiments were either performed in triplicates (S585 and S588) or duplicates (S591), and the given values represent the mean and standard deviation:

$$
I_{\text {rel }}=\frac{F_{\mathrm{c}} \times I_{\text {phos }}}{F_{\mathrm{c}} \times I_{\text {phos }}+I_{\text {nophos }}}
$$

where $I_{\text {rel }}$ is the relative intensity of peptide with higher phosphorylation state, $\mathrm{Fc}$ is the correction factor, $I_{\text {phos }}$ is the measured intensity of peptide with higher 
phosphorylation state and $I_{\text {nophos }}$ is the measured intensity of peptide with lower phosphorylation state.

Fluorescence anisotropy. Fluorescence anisotropy (FP) was measured with a linear dilution series of CK1 $\delta$ from 0 to $250 \mu \mathrm{M}$ and a constant peptide concentration of $500 \mathrm{nM}$. The measurement was performed with a Tecan Spark plate reader at $25^{\circ} \mathrm{C}$ in 384-well plates with a total assay volume of $10 \mu \mathrm{l}$. The peptide sequence was altered to contain an additional C-terminal cysteine for ATTO488 fluorophore NHS-ester (Attotec) labeling. The fluorophore labeling was performed according to the manufacturer's instructions. The required different phosphorylation states were obtained as described above.

Statistic and reproducibility. Whole ovary microscopy data are presented as mean \pm s.d. ( $n=3$ individual ovaries from different animals). All FP experiments were conducted as triplicates, and values given in Supplementary Table 3 represent the mean \pm s.d. $K_{\mathrm{M}} / v_{\max }$ determination was done in triplicates for S585 and S588 phosphorylation and as a duplicate for S591 phosphorylation; values shown in Supplementary Table 5 represent mean \pm s.d. Each data point shown in Fig. $6 \mathrm{~b}$ and Extended Data Fig. 5b was spotted four times (technical replicates).

All NMR experiments measuring peptide kinetics were performed at least twice under identical conditions. NMR experiments with full-length TAp63 $\alpha$ were performed once at a 1:1,000 kinase:protein ratio and once at 1:100.

Reporting Summary. Further information on research design is available in the Nature Research Reporting Summary linked to this Article.

\section{Data availability}

All data are fully available upon request. PDB accession codes for the three crystal structures are 6RU6, 6RU7 and 6RU8.

\section{References}

40. Rossi, V. et al. LH prevents cisplatin-induced apoptosis in oocytes and preserves female fertility in mouse. Cell Death Differ. 24, 72-82 (2017).

41. Pampaloni, F., Stelzer, E. H. K. \& Mattheyer, C. Capillary cell, arrangement and method for accommodating, positioning and examining a microscopic specimen. US patent 20150211981A1 (2015).

42. Lohr, F., Gebel, J., Henrich, E., Hein, C. \& Dotsch, V. Towards complete polypeptide backbone NH assignment via combinatorial labeling. J. Magn Reson. 302, 50-63 (2019).

43. Gil, S. et al. NMR spectroscopic studies of intrinsically disordered proteins at near-physiological conditions. Angew. Chem. Int. Ed. 52, 11808-11812 (2013).

44. Bermel, W. et al. Complete assignment of heteronuclear protein resonances by protonless NMR spectroscopy. Angew. Chem. Int. Ed. 44, 3089-3092 (2005).

45. McIntosh, L. P. et al. Detection and assignment of phosphoserine and phosphothreonine residues by ${ }^{13} \mathrm{C}-{ }^{31} \mathrm{P}$ spin-echo difference $\mathrm{NMR}$ spectroscopy. J. Biomol. NMR 43, 31-37 (2009).

46. Kabsch, W. XDS. Acta Crystallogr. D Biol. Crystallogr. 66, 125-132 (2010).

47. Evans, P. R. \& Murshudov, G. N. How good are my data and what is the resolution? Acta Crystallogr. D Biol. Crystallogr. 69, 1204-1214 (2013).

48. McCoy, A. J. Acknowledging errors: advanced molecular replacement with phaser. Methods Mol. Biol. 1607, 421-453 (2017).

49. Long, A. M., Zhao, H. \& Huang, X. Structural basis for the potent and selective inhibition of casein kinase 1 epsilon. J. Med. Chem. 55, 10307-10311 (2012).

50. Emsley, P. Tools for ligand validation in COOT. Acta Crystallogr. D Struct. Biol. 73, 203-210 (2017)

51. Skubak, P., Murshudov, G. N. \& Pannu, N. S. Direct incorporation of experimental phase information in model refinement. Acta Crystallogr. D Biol. Crystallogr. 60, 2196-2201 (2004).

52. Sali, A. \& Blundell, T. L. Comparative protein modelling by satisfaction of spatial restraints. J. Mol. Biol. 234, 779-815 (1993).
53. Humphrey, W., Dalke, A. \& Schulten, K. VMD: visual molecular dynamics. J. Mol. Graph. 14, 27-28 (1996).

54. Bekker, H. et al. GROMACS-a parallel computer for molecular-dynamics simulations. Phys. Comput. 92, 252-256 (1993).

55. Best, R. B. \& Hummer, G. Optimized molecular dynamics force fields applied to the helix-coil transition of polypeptides. J. Phys. Chem. B 113, 9004-9015 (2009).

56. Lindorff-Larsen, K. et al. Improved side-chain torsion potentials for the Amber ff99SB protein force field. Proteins 78, 1950-1958 (2010).

57. Mamatkulov, S. \& Schwierz, N. Force fields for monovalent and divalent metal cations in TIP3P water based on thermodynamic and kinetic properties. J. Chem. Phys. 148, 074504 (2018).

58. Meagher, K. L., Redman, L. T. \& Carlson, H. A. Development of polyphosphate parameters for use with the AMBER force field. J. Comput. Chem. 24, 1016-1025 (2003)

59. Homeyer, N., Horn, A. H. C., Lanig, H. \& Sticht, H. AMBER force-field parameters for phosphorylated amino acids in different protonation states: phosphoserine, phosphothreonine, phosphotyrosine and phosphohistidine. J. Mol. Model. 12, 281-289 (2006).

60. Greis, K. D. et al. MALDI-TOF MS as a label-free approach to rapid inhibitor screening. J. Am. Soc. Mass Spectrom. 17, 815-822 (2006).

61. Heap, R. E. et al. Identifying inhibitors of inflammation: a novel high-throughput MALDI-TOF screening assay for salt-inducible kinases (SIKs). SLAS Discov. 22, 1193-1202 (2017).

\section{Acknowledgements}

We thank I. Theofel, S. Young and E. Chih-Chao Liang for their review of and input into this manuscript. The research was funded by the DFG (DO 545/18-1), the Centre for Biomolecular Magnetic Resonance (BMRZ) and the Cluster of Excellence Frankfurt (Macromolecular Complexes). M.T. was supported by a Fellowship from the fund of the German Chemical Industry. L.S. and G.H. were supported by the Max Planck Society. F.P., K.H. and E.H.K.S. thank the EU Horizon2020 project LSFM4LIFE (grant no. 668350-2) and the ZonMw-BMBF joint sponsored project 'The Onconoid Hub' (grant no. 114027003) for funding. The Structural Genomics Consortium is a registered charity (no. 1097737) that receives funds from the Canadian Institutes for Health Research, the Canadian Foundation for Innovation, Genome Canada through the Ontario Genomics Institute, GlaxoSmithKline, Karolinska Institute, the Knut and Alice Wallenberg Foundation, the Ontario Innovation Trust, the Ontario Ministry for Research and Innovation, Merck \& Co., the Novartis Research Foundation, the Swedish Agency for Innovation Systems, the Swedish Foundation for Strategic Research and the Wellcome Trust.

\section{Author contributions}

J.G. and M.T. performed NMR and kinetic assays in vitro and in ovaries. A.C. crystallized and solved the structures of the kinase-peptide complexes. K.H. and F.P. performed microscopy experiments and quantitative semi-automated segmentation. L.S. carried out MD simulations. F.L. performed NMR experiments. N.G. measured tetramerization kinetics. F.F., E.H. and J.M. expressed and purified proteins. M.S. measured phosphorylation kinetics. R.L. helped to analyze the data. M.T., G.H., E.H.K.S., S.K. and V.D. designed experiments and analyzed data. J.G., M.T., A.C. and V.D. wrote the manuscript.

\section{Competing interests}

The authors declare no competing interests.

\section{Additional information}

Extended data is available for this paper at https://doi.org/10.1038/s41589-020-0600-3. Supplementary information is available for this paper at https://doi.org/10.1038/ s41589-020-0600-3.

Correspondence and requests for materials should be addressed to M.T. or V.D.

Reprints and permissions information is available at www.nature.com/reprints. 

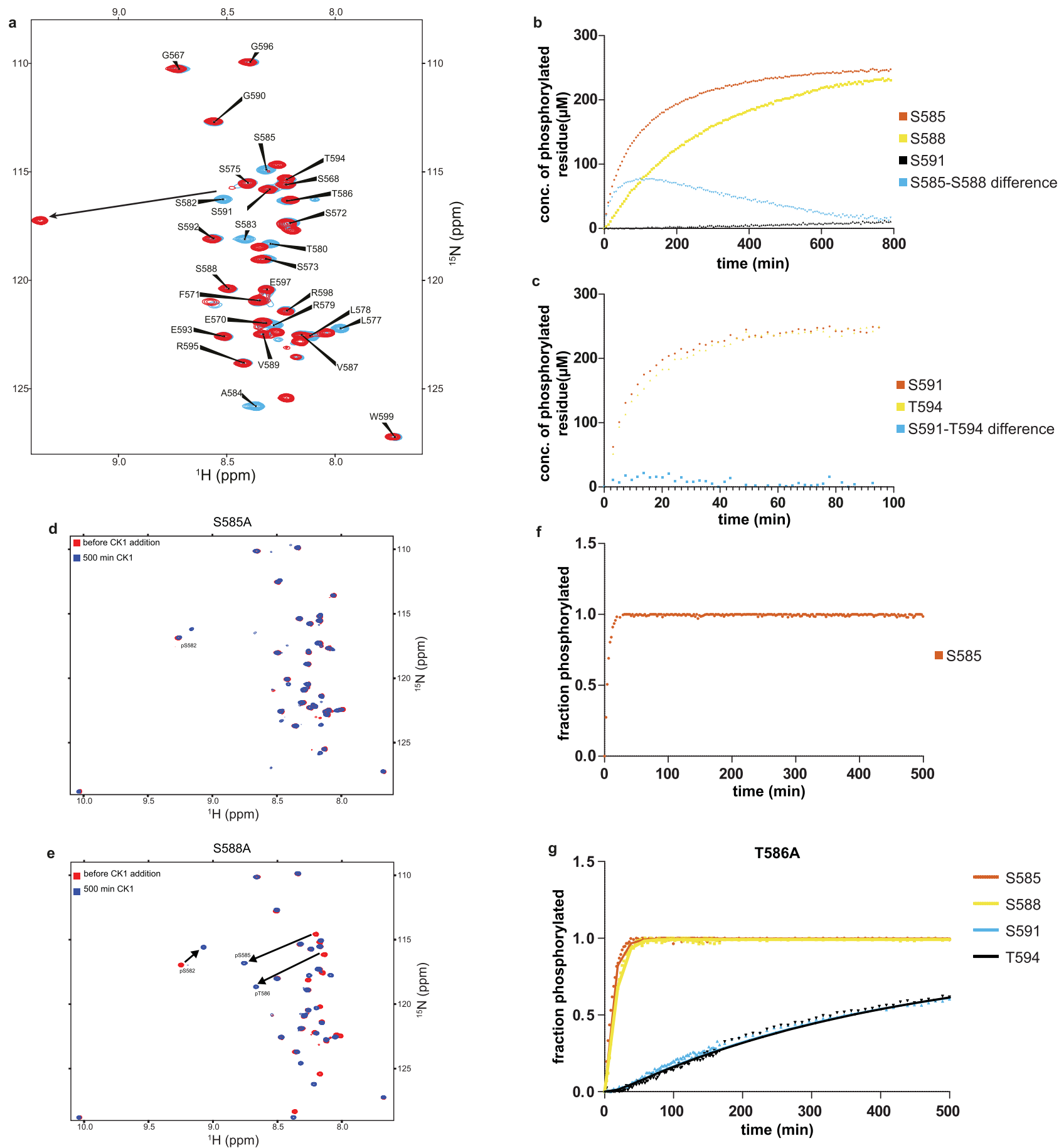

Extended Data Fig. 1 | Phosphorylation kinetics of the PAD peptide. Phosphorylation kinetics of the PAD peptide. a, Overlay of HSQC spectra of unphosphorylated (cyan) and MK2 phosphorylated PAD peptide (red). A large chemical shift change in S582 can be observed as a result of phosphorylation. b. Phosphorylation kinetics of S582 pre-phosphorylated PAD peptide ( $250 \mu \mathrm{M})$ and $12.5 \mathrm{nM}$ CK1 kinase. The phosphorylation of S585 (red) is faster compared to the S588 phosphorylation (yellow) to the extent that the pS582/pS585 intermediate state (cyan) is populated to >1000x of the kinase concentration. c, Phosphorylation kinetics of S582, S585, and S588 pre-phosphorylated PAD peptide ( $250 \mu \mathrm{M})$ with $2.5 \mu \mathrm{M}$ CK1 kinase. The phosphorylation of S591 (red) is faster than T594 (yellow). The difference is smaller, but the concentration of the intermediate state is at least $4 \mathrm{x}$ larger than the kinase concentration. $\mathbf{d}$, Overlay of $[15 \mathrm{~N}, 1 \mathrm{H}]-\mathrm{HSQC}$ spectra of a pS582/S585A PAD peptide (red) and the same peptide after 500 min of exposure to CK1 (blue, 1:2000 kinase:substrate ratio, $25^{\circ} \mathrm{C}$ ). Only partial phosphorylation of a single residue in the vicinity of S582 is visible, indicated by the splitting of the signal, accounting for $45 \%$ of the population of S582. e, f, Overlay of [15 N, 1H]-HSQC spectra of a pS582/S588A PAD peptide (red) and the same peptide after 500 min of exposure to CK1 and phosphorylation kinetics, demonstrating that S585 and T586 are the only residues phosphorylated by CK1 when the second phosphorylation site for CK1 is eliminated. g, Mutation of T586 to alanine does not change the biphasic behavior of the CK1 phosphorylation of the PAD peptide. All experiments were repeated twice and reproducible. Only one replicate is shown in each case. 
a
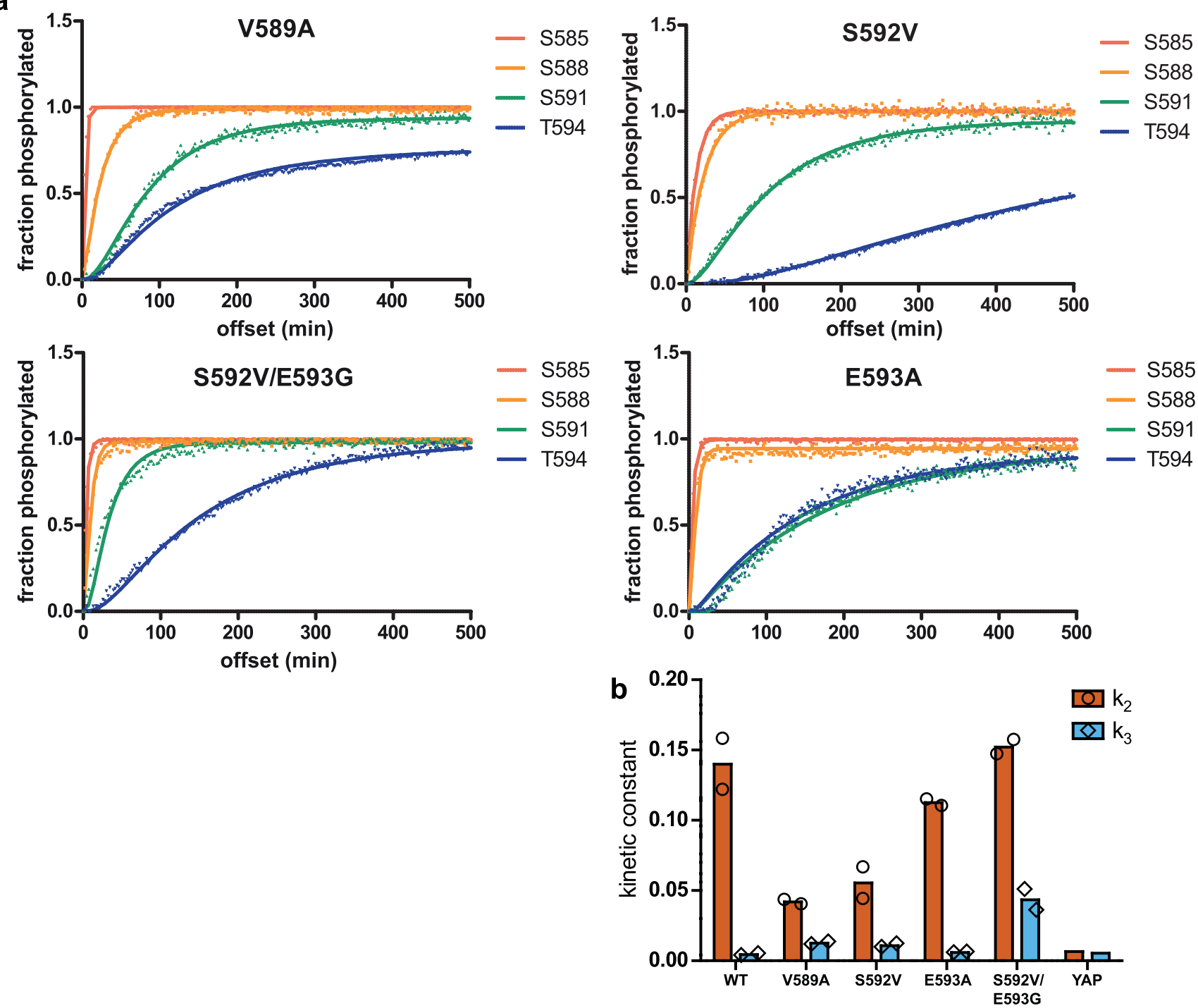

Extended Data Fig. 2 | Phosphorylation kinetics of PAD peptide mutants. Phosphorylation kinetics of PAD peptide mutants. a, Phosphorylation kinetics of selected PAD mutants showing a reduction in the kinetic difference between S588 and S591. b, Measured kinetic constants for the phosphorylation of S588 (k2) and S591 (k3) in wild type and different mutant PAD peptides as well as the constants for the second and third phosphorylation event in the YAP1 peptide. Measurements were repeated twice under identical conditions. Individual data points are shown. The height of the bar represents the mean value and single measurements are indicated. The phosphorylation kinetics of YAP was measured once. 
a

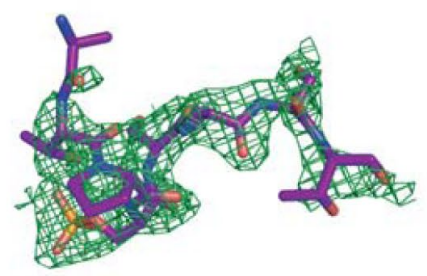

PAD-1P b

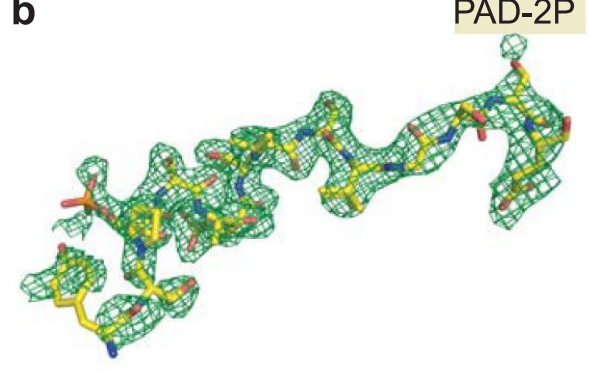

PAD-3P

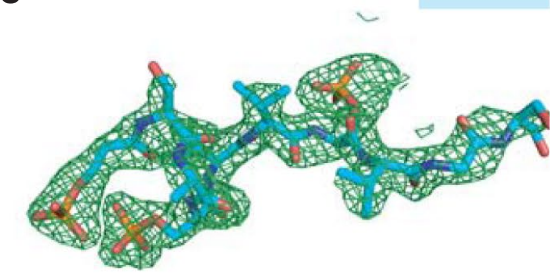

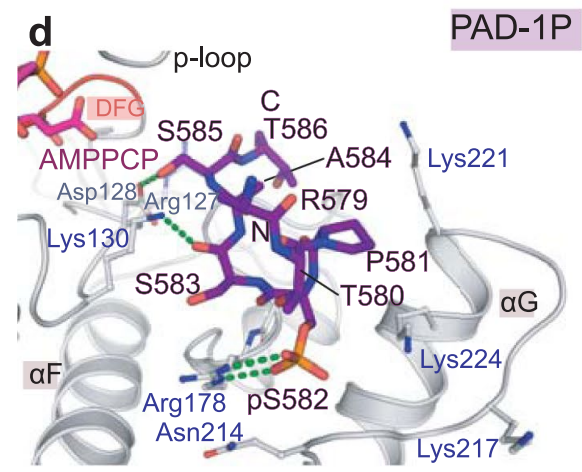
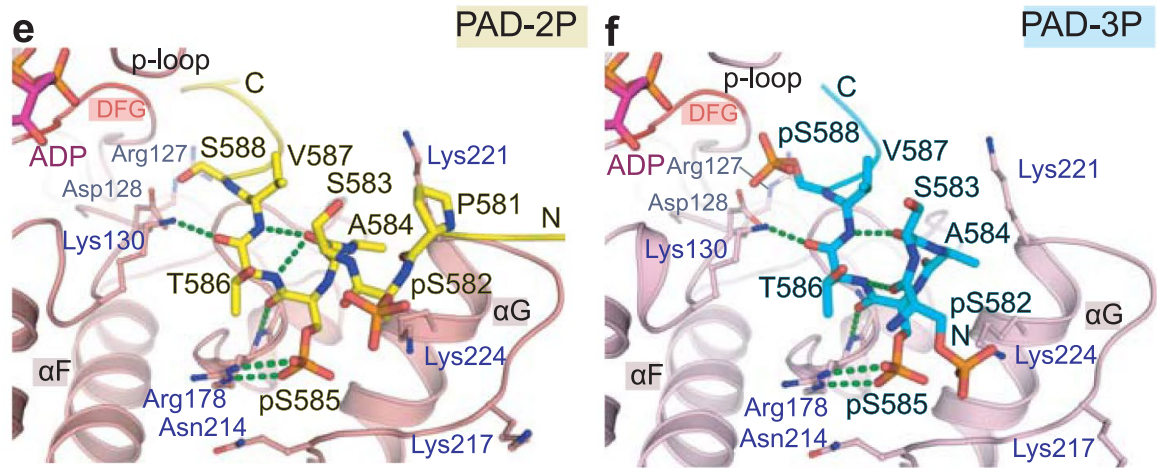

Extended Data Fig. 3 | Crystallographic details. Crystallographic details. a-c, Binding of the PAD peptides within CK16 in the crystal structures. |Fo |-|Fc| omitted electron density map contoured at three-sigma for the bound PAD peptides. d-f, Detailed interactions at the N termini of the PAD peptides within the kinase. 
a

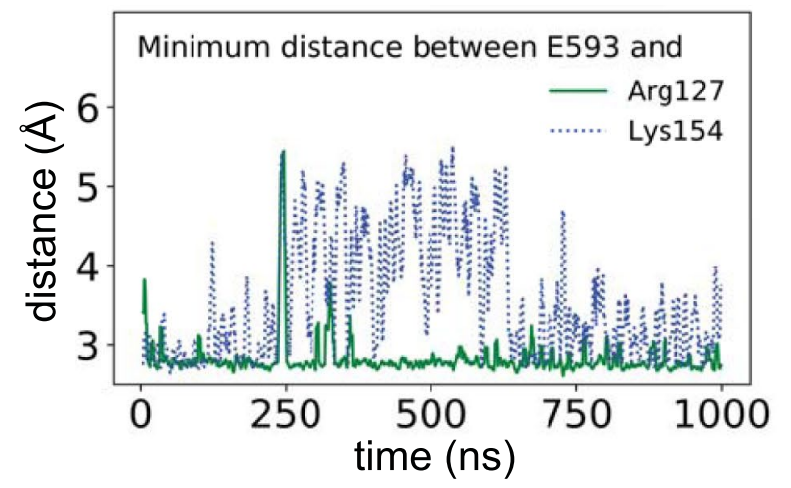

$c$

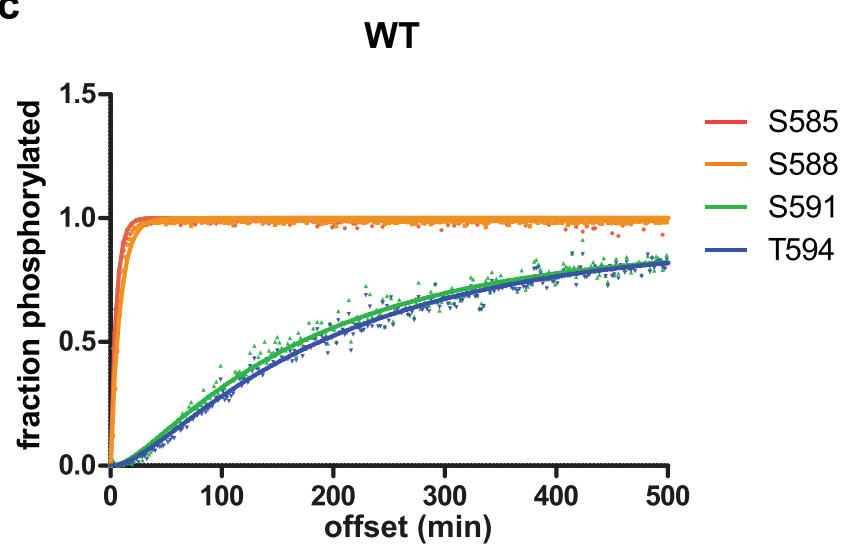

Lys154Glu

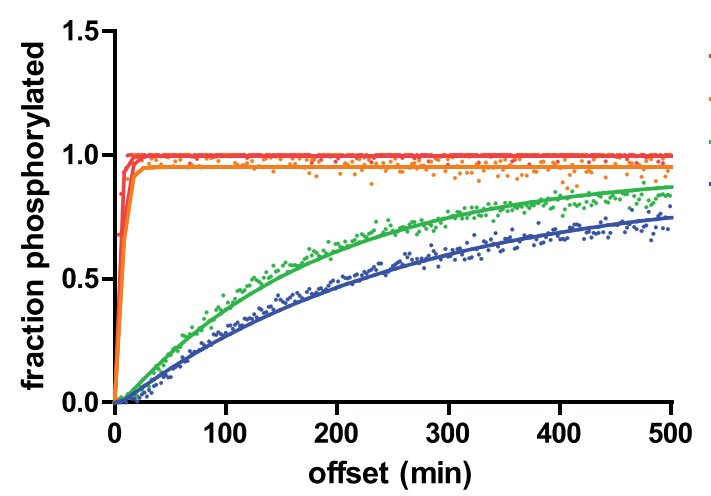

b

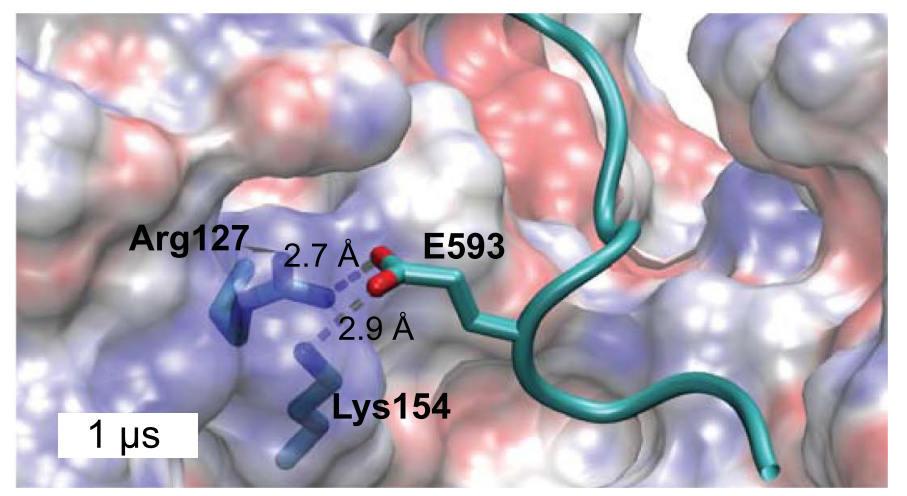

Arg127Glu

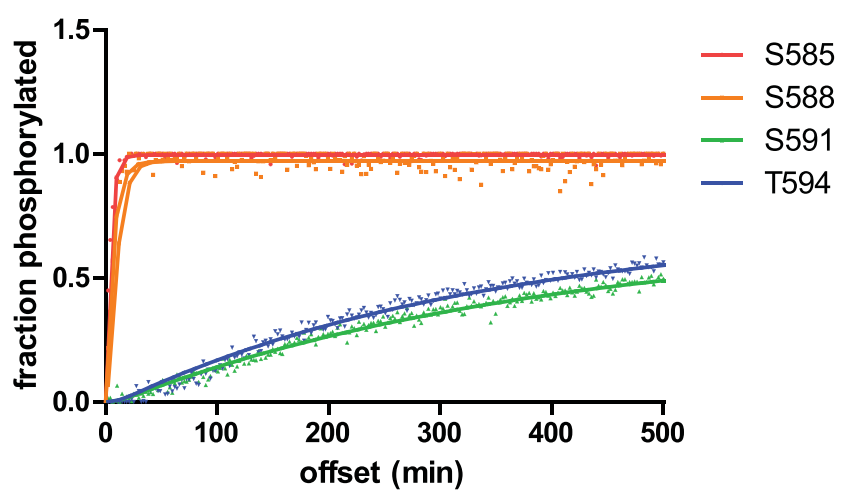

Lys171Glu

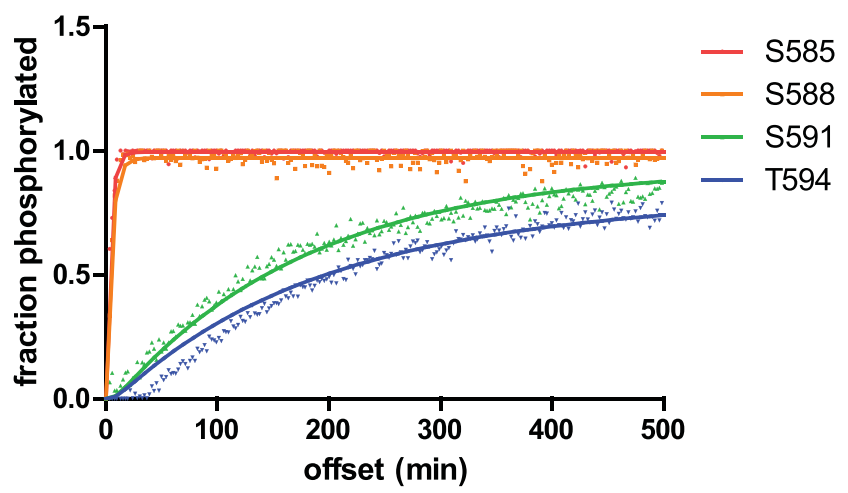

Extended Data Fig. 4 | MD simulation and kinetics with CK1 mutants. MD simulation and kinetics with CK1 mutants. a, MD simulation of CK1 in complex with a shorter PAD-3P peptide (ACE-TPpSSApSTVPSVGSSETRG-NME) with N-terminal acetyl and C-terminal methylamino capping groups showing similar results as the longer peptide (Fig. 5a, b). b. Snapshot at $1 \mu$ s, zooming in on the C-terminal region of the shorter p63 peptide. CK1 is shown as a transparent electrostatic surface (blue/red for positive/negative charge) and the p63 peptide is represented as a cyan cartoon. The residues E593, Arg127 and Lys154 are highlighted. The minimum distances between E593 and the basic residues are indicated. c, Phosphorylation kinetics of wild type and selected CK18 mutants showing a reduction in the kinetic difference between S588 and S591 compared to the wild type kinase for Lys171Glu and Lys154Glu mutants. For Arg127Glu the kinetics is, however, slower. CK1 amino acids are labeled in three-letter code and PAD residues are labeled in one-letter code. All kinetic experiments involving kinase mutants were measured once. 
a

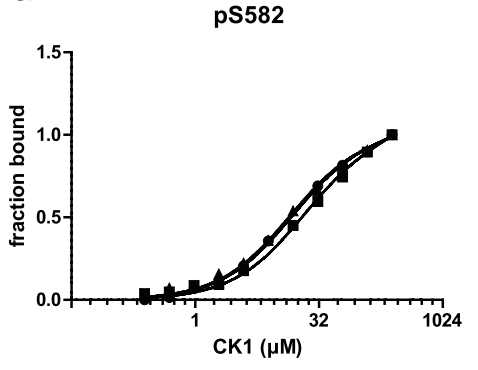

b

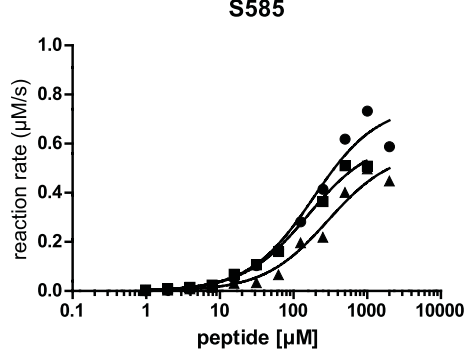

pS582, pS585

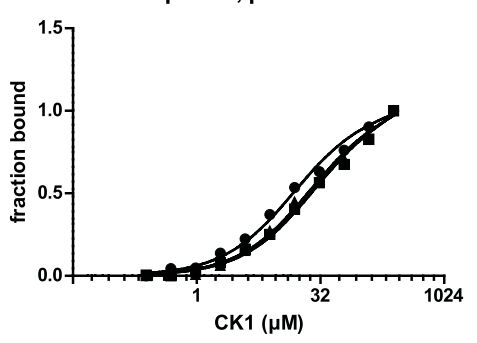

S588

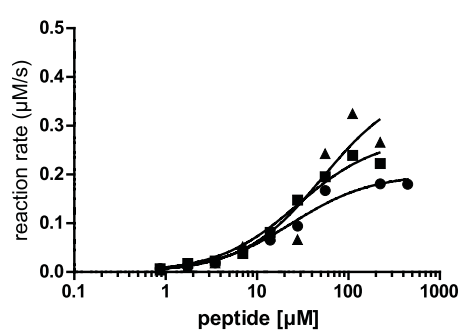

pS582, pS585, pS588

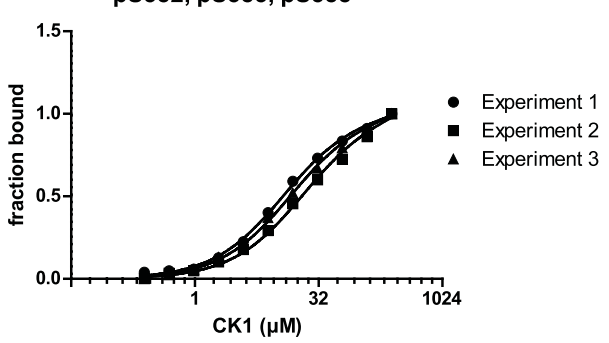

S591

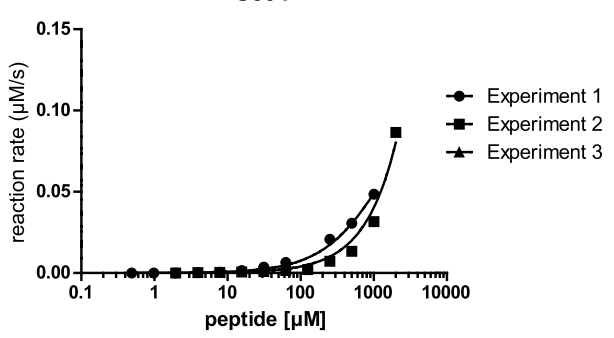

C

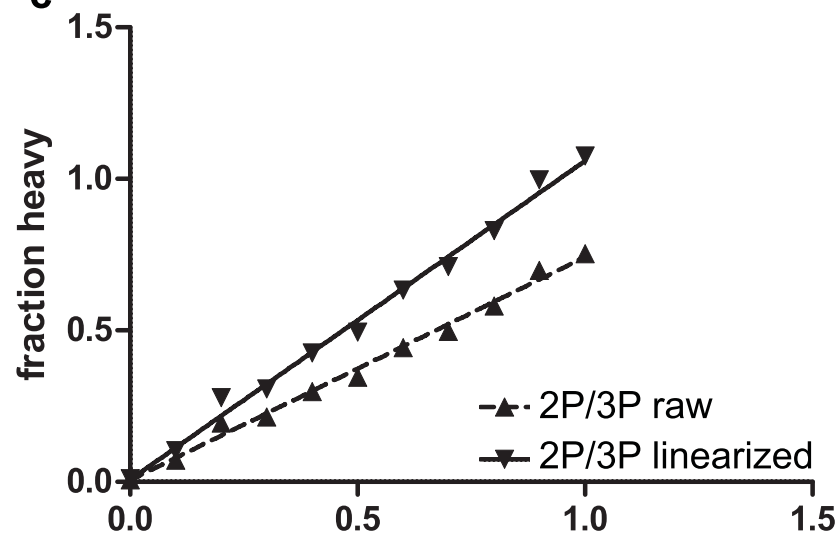

e

e molar ratio d

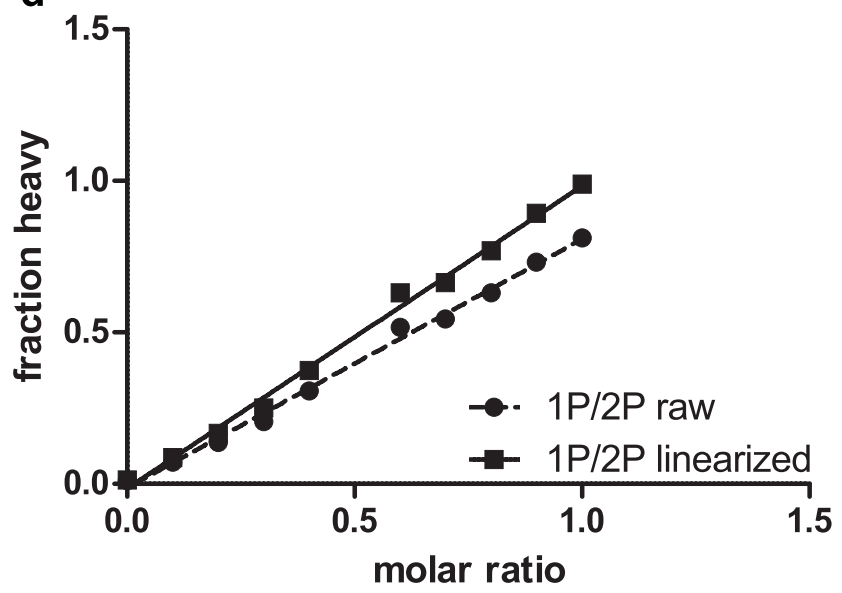

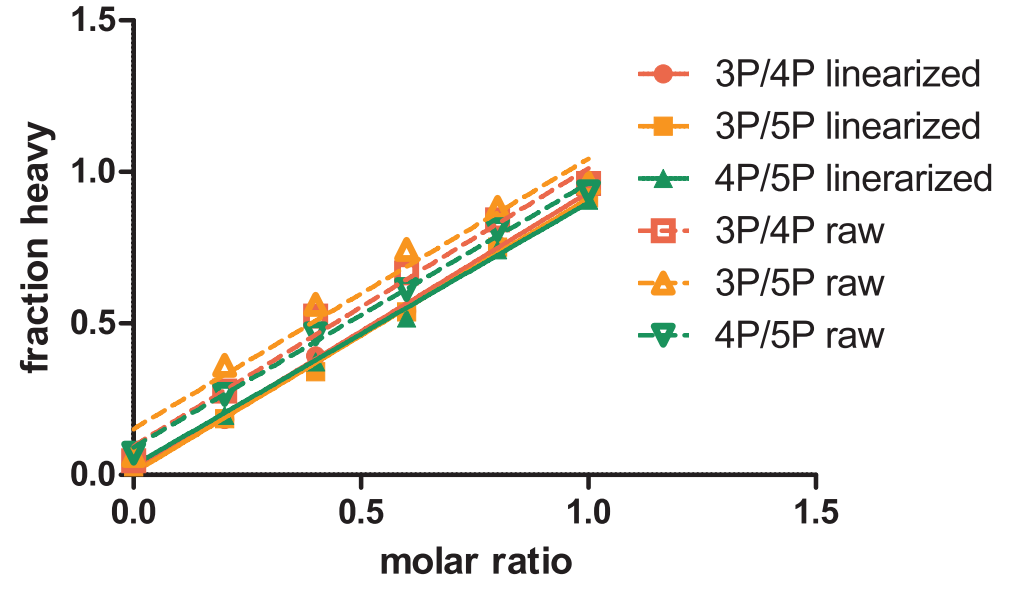

Extended Data Fig. 5 | Measurements of binding affinity and enzyme kinetics. Measurements of binding affinity and enzyme kinetics. a, Three independent KD measurements for each phosphorylation state of the peptide are shown (see also Fig. 6a and Supplementary Table 3). Values given in

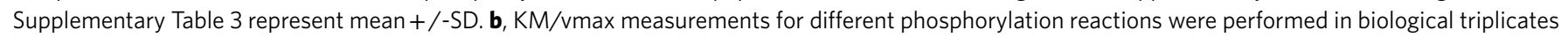
for S585 as well as S588 and duplicates for S591. In all subpanels single experiments are shown individually (see also Fig. 6 b and Supplementary Tables 4 and 5). Values given in Supplementary Table 5 represent mean +/-SD. c-e, Linearization curves to account for nonlinear ionization behavior of PAD peptides with different phosphorylation states. Linearization curves were determined once. 


\section{Reporting Summary}

Nature Research wishes to improve the reproducibility of the work that we publish. This form provides structure for consistency and transparency in reporting. For further information on Nature Research policies, see our Editorial Policies and the Editorial Policy Checklist.

\section{Statistics}

For all statistical analyses, confirm that the following items are present in the figure legend, table legend, main text, or Methods section.

$\mathrm{n} / \mathrm{a}$ Confirmed

$\bigotimes$ The exact sample size $(n)$ for each experimental group/condition, given as a discrete number and unit of measurement

$\bigotimes$ A statement on whether measurements were taken from distinct samples or whether the same sample was measured repeatedly

The statistical test(s) used AND whether they are one- or two-sided

Only common tests should be described solely by name; describe more complex techniques in the Methods section.

$\bigotimes \square$ A description of all covariates tested

Х $\square$ A description of any assumptions or corrections, such as tests of normality and adjustment for multiple comparisons

A full description of the statistical parameters including central tendency (e.g. means) or other basic estimates (e.g. regression coefficient) AND variation (e.g. standard deviation) or associated estimates of uncertainty (e.g. confidence intervals)

For null hypothesis testing, the test statistic (e.g. $F, t, r$ ) with confidence intervals, effect sizes, degrees of freedom and $P$ value noted

Х $\square$ Give $P$ values as exact values whenever suitable.

Х $\square$ For Bayesian analysis, information on the choice of priors and Markov chain Monte Carlo settings

$\bigotimes \square$ For hierarchical and complex designs, identification of the appropriate level for tests and full reporting of outcomes

$\triangle \square$ Estimates of effect sizes (e.g. Cohen's d, Pearson's $r$ ), indicating how they were calculated

Our web collection on statistics for biologists contains articles on many of the points above.

\section{Software and code}

Policy information about availability of computer code

Data collection Topspin (Bruker9; VMD Molefracture, Modeller, Gromacs

Data analysis FUJ, Topspin (Bruker), Sparky, Phaser, Coot, REFMAC, Molprobity and Prism 8.1

For manuscripts utilizing custom algorithms or software that are central to the research but not yet described in published literature, software must be made available to editors and

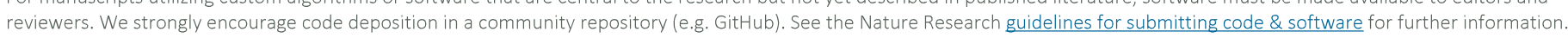

\section{Data}

Policy information about availability of data

All manuscripts must include a data availability statement. This statement should provide the following information, where applicable:

- Accession codes, unique identifiers, or web links for publicly available datasets

- A list of figures that have associated raw data

- A description of any restrictions on data availability

all materials used in this study are commercially available. Accession codees for the three crystal structures are provided in the manuscript. 
Please select the one below that is the best fit for your research. If you are not sure, read the appropriate sections before making your selection.

\ Life sciences

Behavioural \& social sciences

Ecological, evolutionary \& environmental sciences

For a reference copy of the document with all sections, see nature.com/documents/nr-reporting-summary-flat.pdf

\section{Life sciences study design}

All studies must disclose on these points even when the disclosure is negative.

Sample size in general we have performed experiments in triplicate unless specifically indicated
Data exclusions no data were excluded
Replication all experiments could be reliably reproduced
Randomization in the oocyte fragment culture three biological replicates were performed by culturing 6-8 oocyte fragments from different mice together.
Three out of this were counted and represented in the bargraphs.
Blinding was not performed

\section{Reporting for specific materials, systems and methods}

We require information from authors about some types of materials, experimental systems and methods used in many studies. Here, indicate whether each material, system or method listed is relevant to your study. If you are not sure if a list item applies to your research, read the appropriate section before selecting a response.

\begin{tabular}{|c|c|c|c|}
\hline \multicolumn{2}{|r|}{ Materials \& experimental systems } & \multicolumn{2}{|c|}{ Methods } \\
\hline$n / a$ & Involved in the study & $\mathrm{n} / \mathrm{a}$ & Involved in the study \\
\hline ए & Х Antibodies & Х & ChIP-seq \\
\hline Х & Eukaryotic cell lines & Х & $\square$ Flow cytometry \\
\hline Х & $\square$ Palaeontology and archaeology & $\bigotimes$ & MRI-based neuroimaging \\
\hline
\end{tabular}

$\square$ \ Animals and other organisms

Х Human research participants

\ $\square$ Clinical data

\ $\square$ Dual use research of concern

\section{Antibodies}

Antibodies used

anti p63a (D2K8K XP, Cell Signalling), anti cleaved PARP (D6X6X, Cell Signalling, anti VASA (DDX-4) (ab13840, Abcam) and anti Msy2 (N-13, SCBT)

Validation

no further validation was performed

\section{Animals and other organisms}

Policy information about studies involving animals; ARRIVE guidelines recommended for reporting animal research

Laboratory animals $\quad$ P8 CD-1 mice were used for the ovary culture experiments

Wild animals

were not used

Field-collected samples

were not used

Ethics oversight

The protocol for harvesting mouse ovaries was approved by the Tierschutzbeauftragte of Goethe University Frankfurt/Main

Note that full information on the approval of the study protocol must also be provided in the manuscript. 